\title{
Phase analysis method for burst onset prediction
}

\author{
Flavio Stellino, ${ }^{1, *}$ Alberto Mazzoni, ${ }^{2, \dagger}$ and Marco Storace ${ }^{1, \ddagger}$ \\ ${ }^{1}$ DITEN, University of Genoa, Via Opera Pia 11a, 16145 Genova, Italy \\ ${ }^{2}$ The BioRobotics Institute, Scuola Superiore Sant'Anna, Viale Rinaldo Piaggio 34, 56025 Pontedera, Italy
}

(Received 14 December 2016; published 21 February 2017)

\begin{abstract}
The response of bursting neurons to fluctuating inputs is usually hard to predict, due to their strong nonlinearity. For the same reason, decoding the injected stimulus from the activity of a bursting neuron is generally difficult. In this paper we propose a method describing (for neuron models) a mechanism of phase coding relating the burst onsets with the phase profile of the input current. This relation suggests that burst onset may provide a way for postsynaptic neurons to track the input phase. Moreover, we define a method of phase decoding to solve the inverse problem and estimate the likelihood of burst onset given the input state. Both methods are presented here in a unified framework, describing a complete coding-decoding procedure. This procedure is tested by using different neuron models, stimulated with different inputs (stochastic, sinusoidal, up, and down states). The results obtained show the efficacy and broad range of application of the proposed methods. Possible applications range from the study of sensory information processing, in which phase-of-firing codes are known to play a crucial role, to clinical applications such as deep brain stimulation, helping to design stimuli in order to trigger or prevent neural bursting.
\end{abstract}

DOI: 10.1103/PhysRevE.95.022412

\section{INTRODUCTION}

The activity of the brain cortex can be described as a superimposition of coordinated oscillations of different sets of neurons spanning a broad range of frequencies [1]. In the past two decades the phase of such oscillations, usually measured by global firing rate, local field potential (LFP), or electroencephalogram [2], has been shown to correlate with a variety of brain functions. For instance, studies on communication through coherence [3] suggest that interactions between two areas are possible only when the activities in the two areas have the same phase relatively to the appropriate frequency band. Surely the phase of cortical network subthreshold oscillations (captured by LFP [4]) defines the windows of opportunity for the incoming spikes to trigger a response in the local network, hence it is crucial to regulate information transmission. On the other hand, however, the subthreshold phase is not a neural feature that can be directly exploited for information transmission, since it is invisible to the other neurons unless it is associated with firing. It is the relationship between firing and phase that lies at the core of processes such as those observed in the hippocampus [5,6] or the sensory cortex [7-9]. Modeling studies have proved that stimulus-dependent phase locking of firing can be reproduced with networks of leaky integrate and fire (LIF) neurons [10,11]. However, also to ensure efficacy on postsynaptic neurons, in some neural structures such as the olfactory bulb [12] and the thalamus [13], neurons tend to fire in a burst of spikes rather than with isolated action potentials. Both the olfactory bulb [14] and the thalamus [15] display indeed a phase locking between burst onsets and input phase that a simple LIF model cannot account for. A recent interesting modeling study [16] considers this locking and suggests that the way the LFP phase is converted into firing is through

\footnotetext{
*flavio.stellino@edu.unige.it

†alberto.mazzoni@sssup.it

${ }_{\ddagger}^{\ddagger}$ marco.storace@unige.it
}

input-phase modulation of burst onset. The ability of bursts to encode the input phase is moreover proved to be qualitatively robust to the specific choice of bursting model [17].

In this paper we introduce and validate a method able to give a complete analytical description of the way burst onset depends on the phase of the input (phase coding) but also to invert the problem and estimate the likelihood of burst onset given the input state (phase decoding). The phase coding part in particular provides a solution to the issue of whether the brain can exploit LFP information. We suggest that burst onset may be a way for postsynaptic neurons to track the LFP phase. The method is tested with three different neuron models able to generate bursts and with different kinds of inputs, including standard sinusoidal inputs but also inputs mimicking the transitions between up and down states [18], since this transition has been shown to depend on the phase of the process $[19,20]$.

\section{METHODS}

The proposed method is structured in three parts. The first part (phase coding) comprises a characterization of the neuron phase coding properties (done by stimulating the neuron with stochastic stimuli with different spectral features and recording the corresponding neuron responses), an analysis of the neuron responses with interburst interval (IBI) detection and extraction of a set of phase profiles (corresponding to the detected IBIs) that cause burst onset, and a consequent identification of the phase coding mechanism for the chosen neuron model.

We point out that this part can be viewed as a specific method per se and can be applied also without a subsequent decoding. Moreover, it allows highlighting the correlation between phase profiles of the input current and burst onset of the membrane potential, which is another element of this paper. The second part of the method is the phase decoding procedure. Finally, the third part is the identification of burst onset probability for a generic input phase profile. 


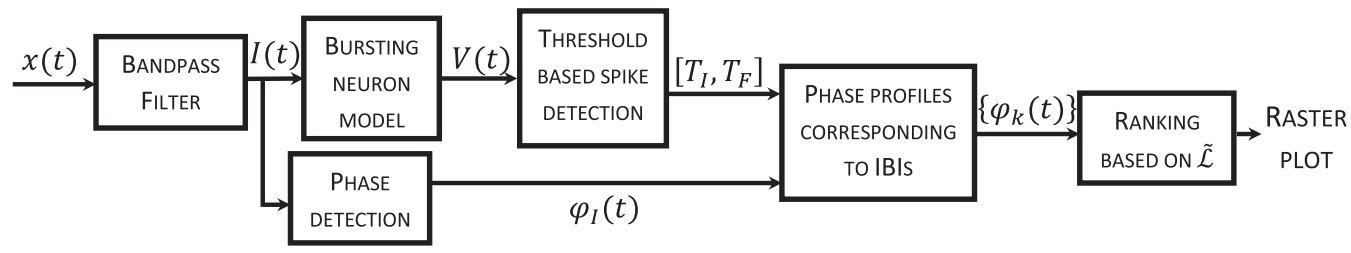

FIG. 1. Block scheme describing at a glance the phase coding part of the method applied to obtain raster plots of the phase profiles. The input signal $x(t)$ is filtered to obtain the current $I(t)$, which is the input of both a two-compartment neuron model with active currents (reduced Traub model) (see Appendix A and [21]) and a phase detection block (see Appendix D). From the neuron output $V(t)$ we extract the spike times and then the IBIs. By combining this information with the phase detected from $I(t)$, we obtain the phase profiles corresponding to IBIs. Finally, these phase profiles are ordered according to their lengths [the vector $\tilde{\mathcal{L}}$ is defined in Eq. (2)] and represented in a raster plot.

The whole method is detailed in the following, by using as reference a pyramidal neuron model (the same considered in [16]) with somatic and dendritic compartments [21], described in detail in Appendix A. In this neuron model, bursting is induced by a persistent sodium current [see Eq. (A5)] and a slow potassium current [see Eq. (A6)] in the dendrite [22].

\section{A. Part 1: Phase coding}

The phase coding part is described at a glance in Fig. 1. Through the methods reported in Appendix B, we generate signals $x(t)$ as realizations of stochastic processes [white noise, pink noise, brown noise, and Ornstein noise (see Appendix B)] characterized by null mean, standard deviation $\sigma_{x}$ (fixed for all processes), and different power spectral densities (PSDs), obtained as described in Appendix C, in the frequency band $[1,21] \mathrm{Hz}$. Then each signal $x(t)$ is filtered through a finite impulse response (FIR) digital filter (of order 500, with Hamming window to reduce the spectral leakage) in partially overlapping frequency windows of bandwidth $4 \mathrm{~Hz}$, i.e., [1,5], [3, 7], [5,9], . . , [17,21] Hz, thus obtaining the synaptic input currents $I(t)$.
The somatic membrane potential $V(t)$ in response to $I(t)$ is computed by integrating the considered model through the explicit Euler method with a fixed step of $20 \mu \mathrm{s}$. The black lines in Figs. 2(a)-2(d) are the four stimuli $I(t)$ (white, pink, Ornstein, and brown noise, respectively), computed by filtering the corresponding signals $x(t)$ (light gray line) in the frequency band [3,7] Hz. The PSDs of the corresponding stochastic processes [computed by Eq. (C1)] are shown in Figs. 2(e)-2(h), both without filtering (light gray lines) and with filtering in the band $[3,7] \mathrm{Hz}$ (black lines).

This first step is necessary to characterize the chosen neuron model through input signals with frequency contents typical for LFPs. The method (besides the example of Fig. 3) requires that the stochastic stimulation is done by using all four of these synaptic inputs, in sequence.

As a second step, through a threshold-based spike-detection method, with a threshold of $-20 \mathrm{mV}$, the interspike intervals (ISIs) are directly computed. From the ISI histogram, it is easy to detect two sets: shorter intraburst ISIs and longer IBIs. The first set is neglected and we focus on the second one.

For the $k$ th IBI $(k=1, \ldots, N)$, we detect the starting time $t_{k}^{I}$ [marked by a gray closed circle in Fig. 3(c)] and the final time

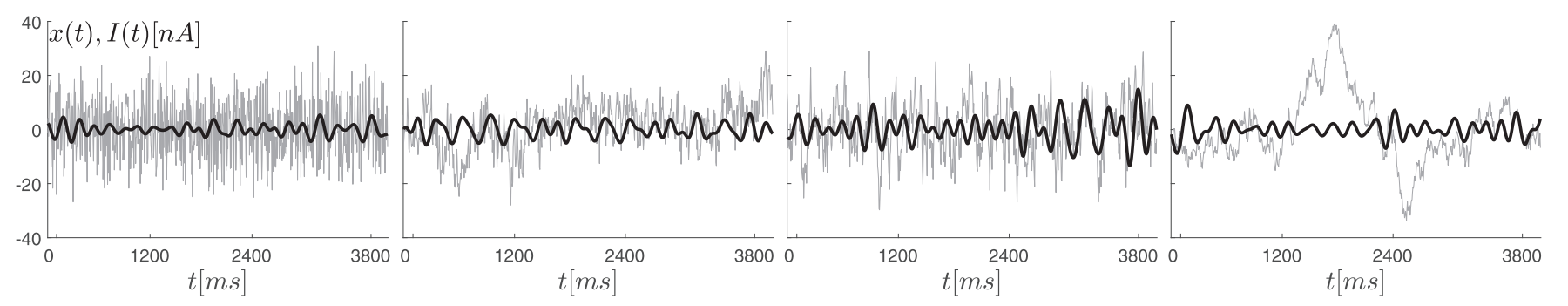

(a)

(c)

(d)

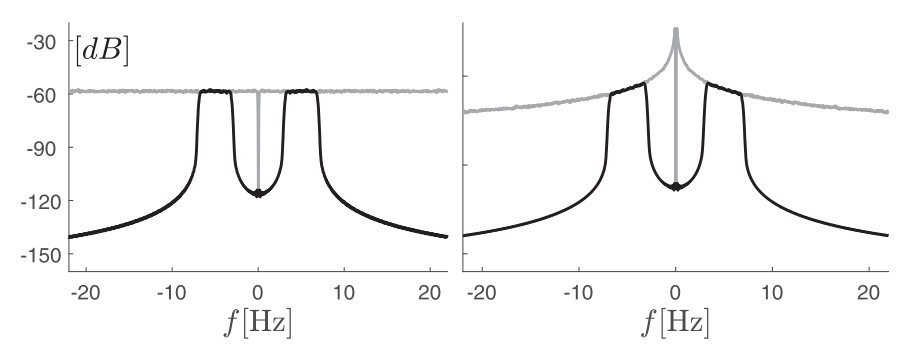

(e)

(f)

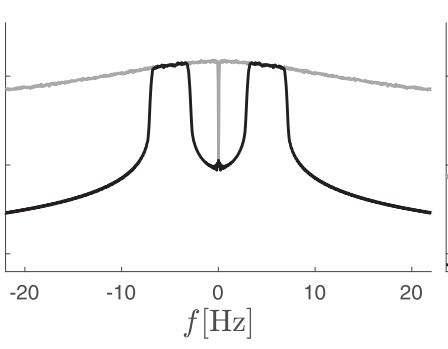

(g)

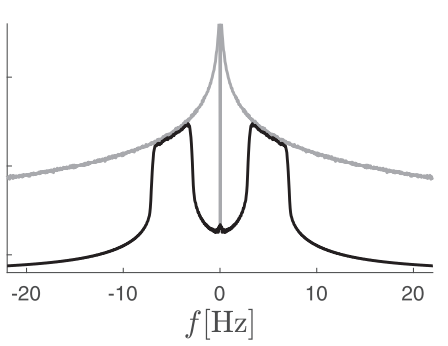

(h)

FIG. 2. (a)-(d) Signals $x(t)$ (light gray lines) and corresponding (after filtering in the frequency band [3,7] Hz) synaptic input currents $I(t)$ (black lines) in nA for white, pink, Ornstein, and brown noise, respectively. (e)-(h) PSDs (in dB) of the corresponding stochastic processes, both without filtering (light gray lines) and with filtering in the band [3,7] Hz (black lines). Since the ordinate axis is common to each row, it is reported only in the leftmost figure. 


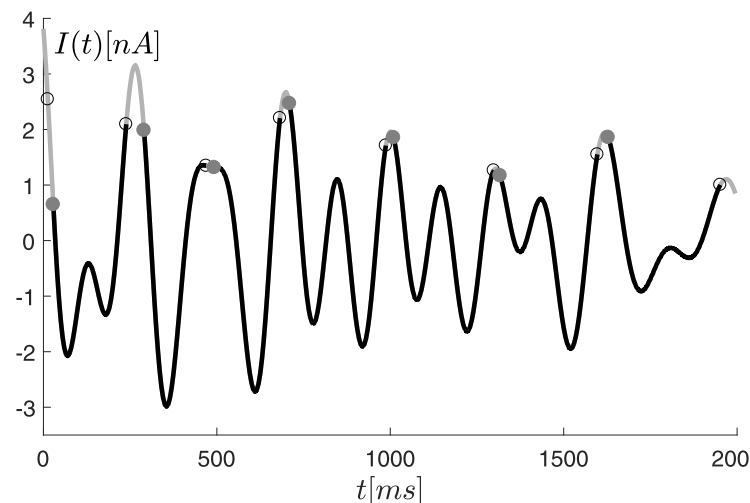

(a)

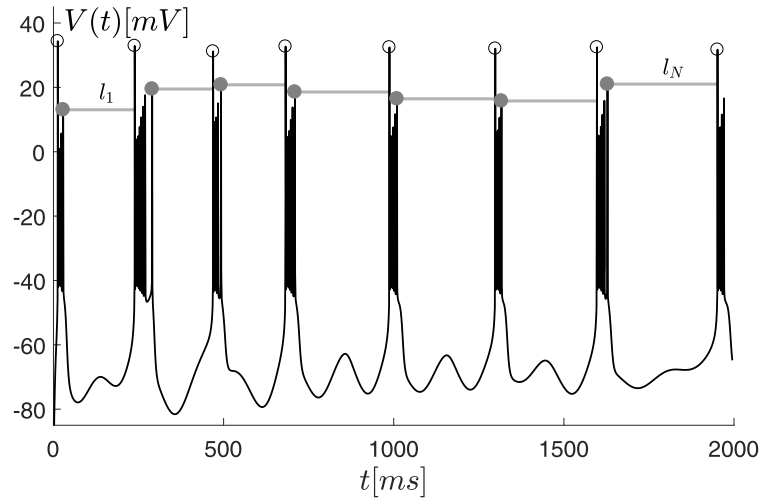

(c)

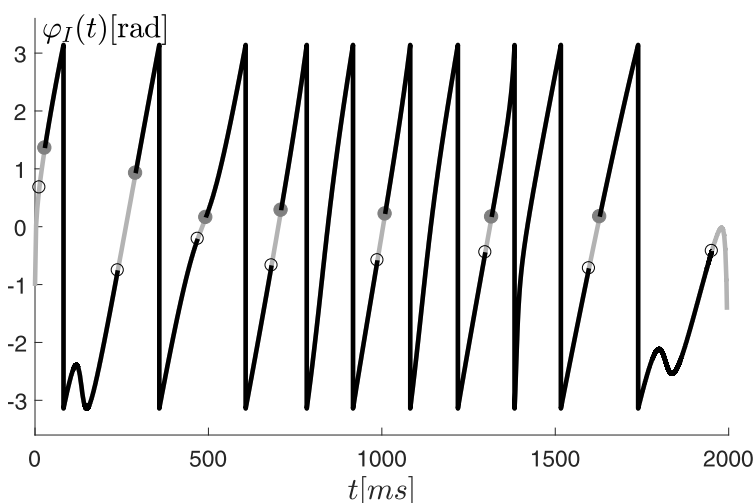

(b)

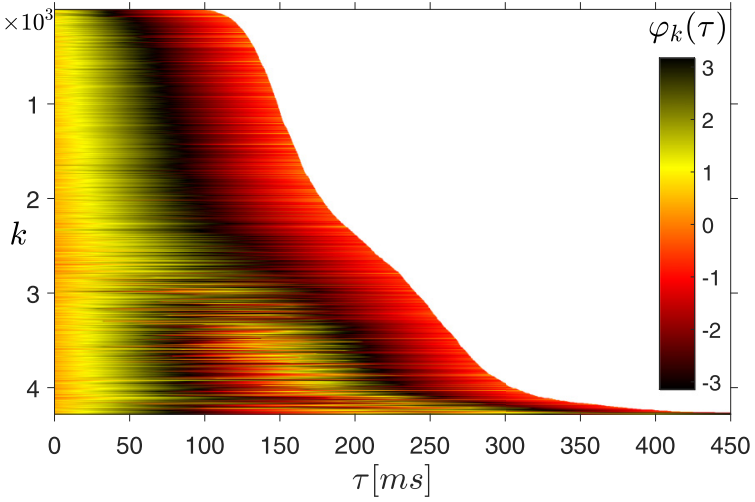

(d)

FIG. 3. Detail of the (a) input stimulus $I(t)$ (white noise filtered in the frequency band $[3,7] \mathrm{Hz}$ ) and (b) corresponding phase $\varphi_{I}(t)$ and (c) membrane potential $V(t)$. The gray closed (open) circles mark the starting (final) point of each IBI. (d) Raster plot of phase profiles $\varphi_{k}(\tau)$, obtained from a simulation of $1000 \mathrm{~s}$. The colors (gray levels) code the phase amplitudes.

$t_{k}^{F}$ (marked by an open circle). These times are stored in two vectors $T_{I}=\left[t_{1}^{I}, \ldots, t_{N}^{I}\right]^{T}$ and $T_{F}=\left[t_{1}^{F}, \ldots, t_{N}^{F}\right]^{T}$. Finally, the vector $\mathcal{L}=T_{F}-T_{I}=\left[l_{1}, \ldots, l_{N}\right]$ contains the IBI lengths $l_{k}(k=1, \ldots, N)$, represented by gray horizontal segments in Fig. 3(c). In the last step of the phase coding method, the phase profile $\varphi_{I}(t)$ of any input stimulus $I(t)$ is detected through the method based on the Hilbert transform described in Appendix D.

The phase profile $\varphi_{k}$ related to the onset of the $k$ th IBI $\left(\left[t_{k}^{I}, t_{k}^{F}\right]\right)$ of length $l_{k}\left(=t_{k}^{F}-t_{k}^{I}\right) \in \mathcal{L}$ is extracted from $\varphi_{I}(t)$ as follows:

$\varphi_{k}\left(t-t_{k}^{I}\right)= \begin{cases}\varphi_{I}(t) & \text { for } t_{k}^{I}<t<t_{k}^{F}, k=1, \ldots, N \\ \text { undefined } & \text { elsewhere. }\end{cases}$

We point out that, at the end of each burst, the neuron memory is reset. For this reason, each phase profile can be expressed as a function of a relative time $\tau\left(=t-t_{k}^{I}\right)$, i.e., as $\varphi_{k}(\tau)$, with $\tau \in\left[0, l_{k}\right]$.

Figure 3(a) shows a detail of an input stimulus $I(t)$ (white noise filtered in the frequency band [3,7] Hz), whose corresponding phase $\varphi_{I}(t)$ is shown in Fig. 3(b). The black pieces of $\varphi_{I}(t)$ are the phase profiles $\varphi_{k}(t)$ in the $k$ th IBI, detected from the corresponding neuronal response, shown in Fig. 3(c). Sorting $\mathcal{L}$ in ascending order, we obtain the ordered vector

$$
\tilde{\mathcal{L}}=\left\{\tilde{l}_{1}, \ldots, \tilde{l}_{N}\right\}
$$

with $\tilde{l}_{j} \leqslant \tilde{l}_{j+1}$. The phase profiles are ordered accordingly. Figure 3(d) shows the raster plot of phase profiles for the considered example. The regular structure of this panel evidences a correlation between the $k$ th IBI length (time length of the nonwhite region for any $k$ ) and the corresponding phase profile [coded by colors (gray levels)], which we use to estimate the phase coding mechanism. The homologous raster plots of phase profiles, obtained with brown noise, Ornstein noise, and pink noise filtered in the frequency band [3,7] $\mathrm{Hz}$ are shown in Figs. 4(a)-4(c), respectively. The three raster plots, as well as the raster plot in Fig. 3(d), have a similar dynamics. For instance, they all display a doubling of the oscillation for IBI lengths higher than about $220 \mathrm{~ms}$.

We assume that the possible IBI lengths are bounded by the edges of $\tilde{\mathcal{L}}$, i.e., they belong to the interval

$$
\Lambda=\left[\tilde{l}_{1}, \tilde{l}_{N}\right]
$$

Let $\mathcal{I}$ be the interval $[0, l]$, with $l \in \Lambda, \Pi$ the circular interval $[-\pi, \pi]$ (see Appendix E), and $\Phi$ the (circular) space of continuous input phase profiles $\varphi: \mathcal{I} \longmapsto \Pi$ that make the neuron activate a burst at $\tau=l \in \Lambda$. Remember that the variable $\tau$ is used to denote the time relative to the beginning of an IBI, i.e., for any IBI of length $l \in \Lambda, \tau \in \mathcal{I}$.

The sorted IBI length vector $\tilde{\mathcal{L}}$ has components belonging (by construction) to the interval $\Lambda$. The corresponding sequence of sorted phase profiles belongs to the functional space $\Phi$. 


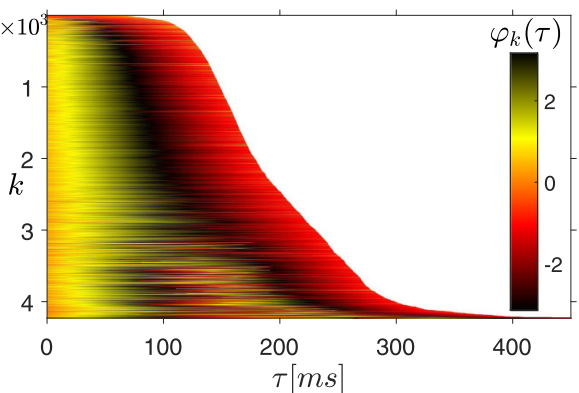

(a)

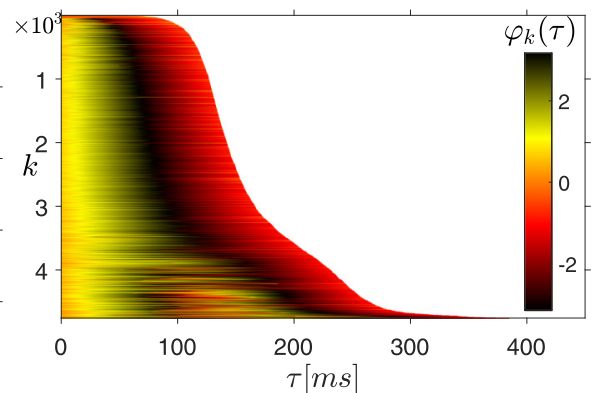

(b)

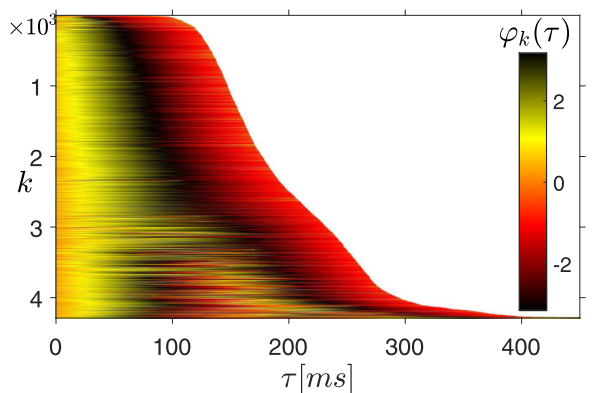

(c)

FIG. 4. Raster plots of phase profiles $\varphi_{k}(\tau)$, obtained from a simulation of $1000 \mathrm{~s}$ with (a) brown noise, (b) Ornstein noise, and (c) pink noise filtered in the frequency band $[3,7] \mathrm{Hz}$. Since the $k$ axis is common to each row, it is shown only in the leftmost figure.

What we call phase coding is nothing but a many-to-one mapping that for any piece of input stimulus of length $l \in$ $\Lambda$ corresponding to an IBI provides the corresponding phase profile $\varphi \in \Phi$. Since the same IBI length can be caused by different stimuli, all these stimuli will be coded by the same phase profile. However, we remark that in order to define a phase decoding, each length $l$ must be one to one associated with a phase profile $\varphi$ (i.e., this specific mapping must be invertible, as detailed in the following section).

\section{B. Part 2: Phase decoding}

The phase decoding procedure is based on the assumption that the phase coding mapping is invertible, i.e., for any $l \in \Lambda$, there exists a unique corresponding phase profile $\varphi(\tau)$. Now we describe how to obtain the decoding mapping from the data stored during the coding procedure. The phase decoding can be expressed in terms of the (bijective) map

$$
\Psi: \Lambda \longmapsto \Phi
$$

which is the inverse of the phase coding mapping and is such that $\Psi[l]=\varphi$.

Let $\lambda_{l}$ be a subinterval of lengths belonging to $\Lambda$, differing at most by $\varepsilon$, and centered around $l$ :

$$
\lambda_{l}=\left[l-\frac{\varepsilon}{2}, l+\frac{\varepsilon}{2}\right], \quad l \in \Lambda .
$$

Assuming that $l$ is a random variable, we can use the histogram of all the lengths $\tilde{l}_{k} \in \lambda_{l}$ to estimate the probability density function (PDF) $f_{l}(\tau)$ of $\lambda_{l}$. Then the mean length of $\lambda_{l}$ can be computed directly from the histogram as the discrete version of the following expression:

$$
\mu_{l}=\int_{\lambda_{l}} \tau f_{l}(\tau) d \tau
$$

Each interval $\lambda_{l}$ maps into the functional subspace $\phi_{l} \subseteq \Phi$, which contains phase profiles $\varphi(t)$ (corresponding to burst onsets) with lengths belonging to $\lambda_{l}$ [see Fig. 5(a)].

For any $\tau, \varphi(\tau)$ is a random variable (defined on the circular interval $\Pi$ ) with time-variant $\operatorname{PDF} f_{l}^{\circ}(\varphi, \tau)$, which can in turn be estimated from the available histograms for any $\tau$. Therefore, any $\varphi \in \phi_{l}$ can be interpreted as a nonstationary process with a time-variant PDF $f_{l}^{\circ}(\varphi, \tau)$ and can be characterized through first-order statistics. The circular mean and circular variance of $f_{l}(\varphi, \tau)$ can be computed as follows (see Appendix E):

$$
\begin{aligned}
& \stackrel{\circ}{\mu}_{l}(\tau)=\arg \left[\int_{\Pi} \stackrel{\circ}{f}_{l}(\varphi, \tau) e^{j \varphi(\tau)} d \varphi\right], \\
& \stackrel{\circ}{l}_{l}^{2}(\tau)=1-\left|\int_{\Pi} \stackrel{\circ}{f}_{l}(\varphi, \tau) e^{j \varphi(\tau)} d \varphi\right|,
\end{aligned}
$$

where $\stackrel{\circ}{\mu}_{l}(\tau)$ is the circular mean phase profile in $\phi_{l}$, with corresponding standard deviation profile $\circ_{l}(\tau) \in[0,1]$. This allows us to define a time-dependent confidence interval of

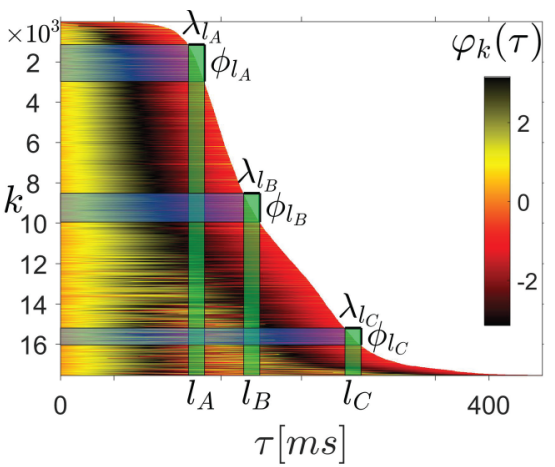

(a)

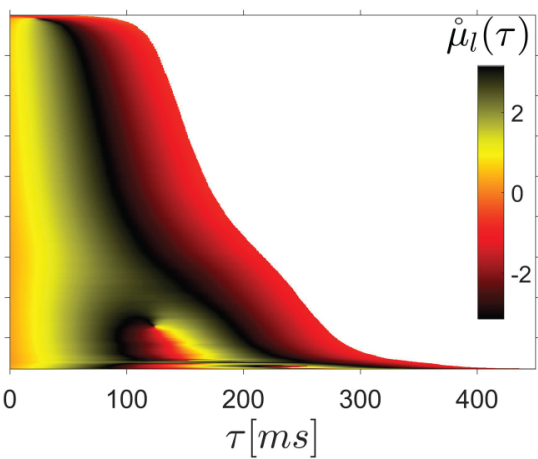

(b)

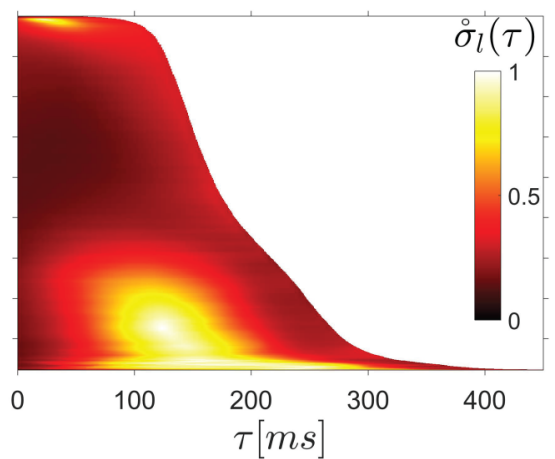

(c)

FIG. 5. (a) Raster plot of the phase profiles $\varphi_{k}$ generated by a stochastic stimulation (comprising all four kinds of stimuli considered) filtered in the band $[3,7] \mathrm{Hz}$ and corresponding raster plots of (b) $\stackrel{\circ}{\mu}_{l}(\tau)$ (map $\Psi$ ) and (c) $\stackrel{\circ}{\sigma}_{l}(\tau)$ (map $\Psi_{\sigma}$ ). Since the $k$ axis is common to each row, it is shown only in the leftmost figure. 


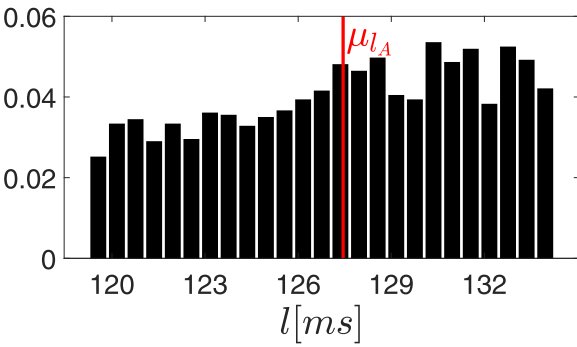

(a)

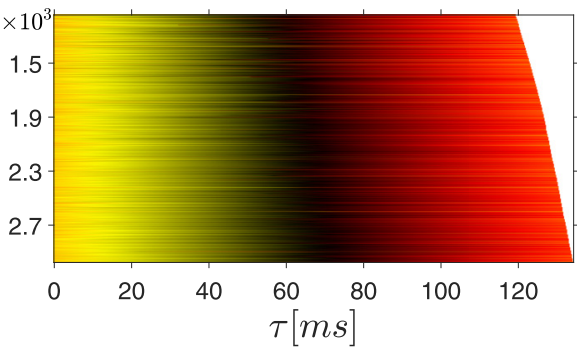

(d)

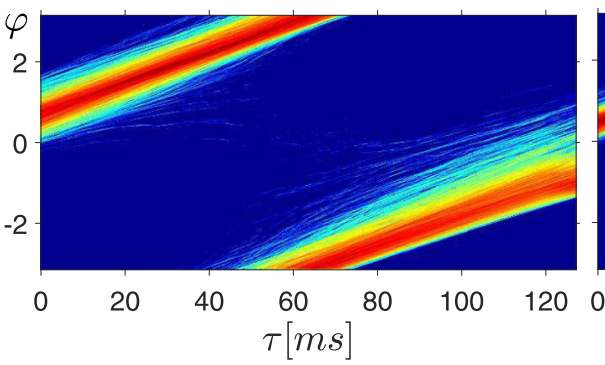

(g)

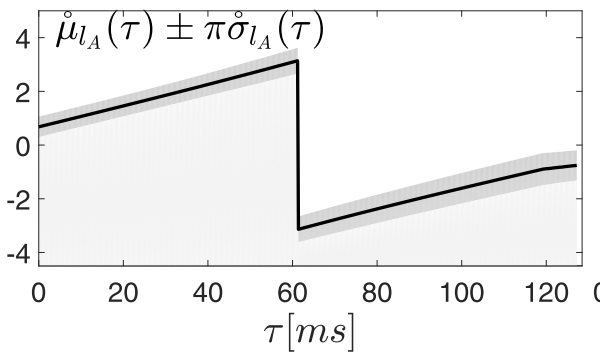

(j)

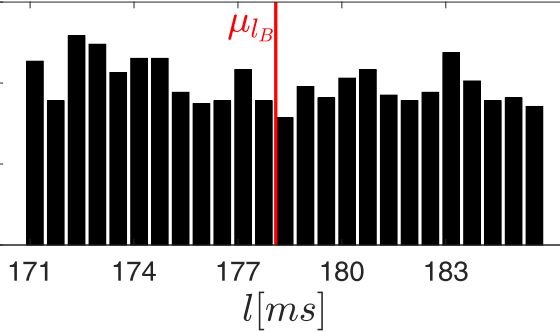

(b)

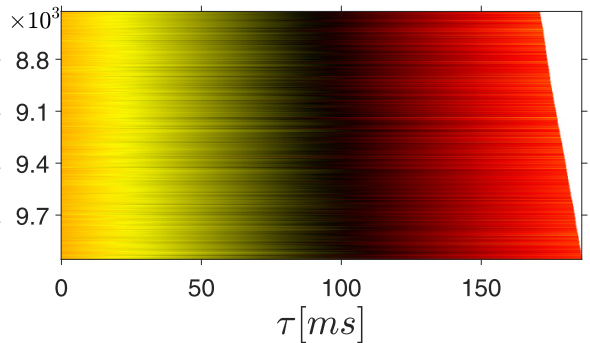

(e)

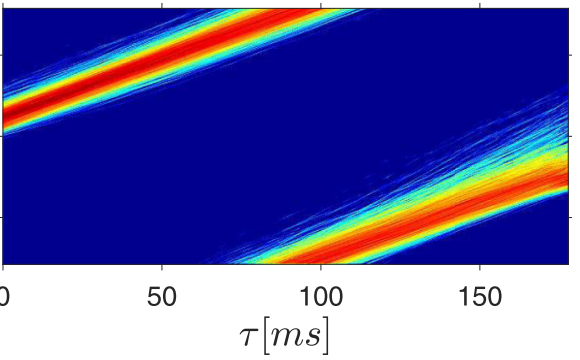

(h)

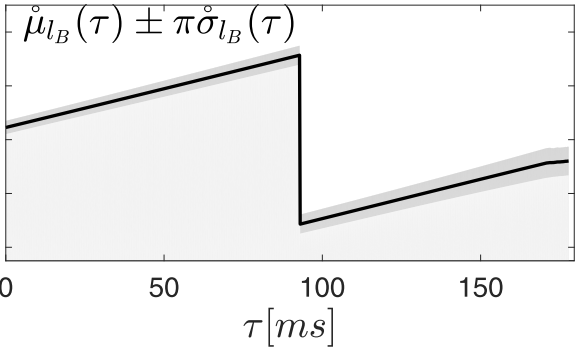

(k)

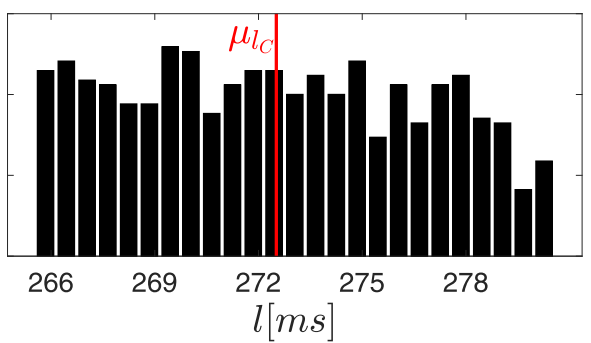

(c)

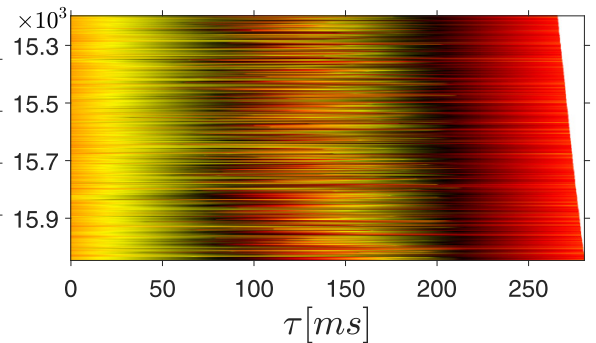

(f)

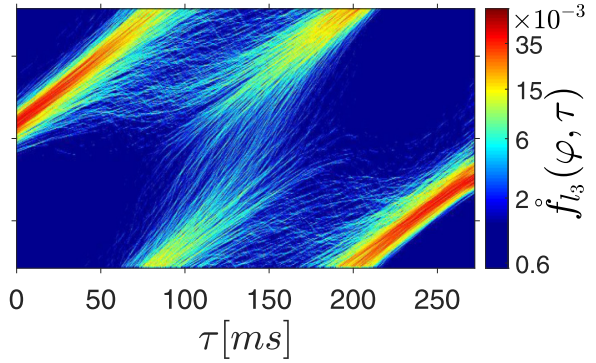

(i)

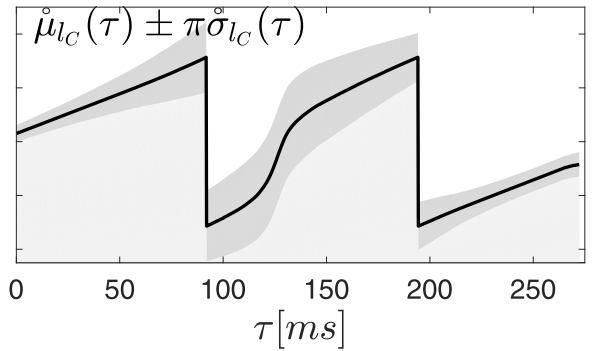

(1)

FIG. 6. Histograms of the IBI lengths contained within the subintervals (a) $\lambda_{l_{A}}$, (b) $\lambda_{l_{B}}$, and (c) $\lambda_{l_{C}}$ [vertical transparent green (light gray) rectangles in Fig. 5(a)]. The mean values $\mu_{l_{A}}, \mu_{l_{B}}$, and $\mu_{l_{C}}$ are marked by vertical red (gray) lines. Also shown is the zoom of the raster plots of phase profiles $\varphi_{k}(t)$ belonging to (d) $\phi_{l_{A}}(k \in[1144,2976])$, (e) $\phi_{l_{B}}(k \in[8524,9955])$, and (f) $\phi_{l_{C}}(k \in[15198,17551])$, highlighted with horizontal transparent blue (gray) rectangles in Fig. 5(a). The corresponding PDFs (g) $\dot{f}_{l_{A}}(\varphi, \tau)$, (h) $f_{l_{B}}(\varphi, \tau)$, and (i) $f_{l_{C}}(\varphi, \tau)$ are shown in logarithmic color (gray level) scale. Also shown are the corresponding circular mean phase profiles (black curves) $(\mathrm{j}) \stackrel{\leftrightarrow}{\mu}_{l_{A}}(\tau),(\mathrm{k}) \stackrel{\circ}{\mu}_{l_{B}}(\tau)$, and (l) ${\stackrel{\circ}{l_{C}}}_{l_{C}}(\tau)$ with confidence intervals (gray regions).

$\pm \pi \stackrel{\circ}{\circ}_{l}$ around $\stackrel{\circ}{\mu}_{l}$ (see Appendix E) to estimate the reliability of $\stackrel{\circ}{l}_{l}(\tau)$ for each $\tau$.

Figure 5(a) shows the raster plot of phase profiles $\varphi_{k}(t)$, extracted from the neural response to stochastic stimulation obtained by using all the four kinds of stimuli considered, filtered in the band $[3,7] \mathrm{Hz}$. Three values $l_{A}, l_{B}$, and $l_{C}$ and corresponding subintervals $\lambda_{l_{A}}, \lambda_{l_{B}}$, and $\lambda_{l_{C}}$ [vertical transparent green (light gray) rectangles] are evidenced, whose histograms are shown in Figs. 6(a)-6(c) with means $\mu_{l_{A}}, \mu_{l_{B}}$, and $\mu_{l_{C}}$ [vertical red (gray) lines]. The raster plots of phase profiles $\varphi_{k}(\tau)$ belonging to $\phi_{l_{A}}, \phi_{l_{B}}$, and $\phi_{l_{C}}$ [highlighted with horizontal transparent blue (gray) rectangles in Fig. 5(a)] are enlarged in Figs. 6(d)-6(f). Figures 6(g)-6(i) show the corresponding PDFs $f_{l_{A}}(\varphi, \tau), f_{l_{B}}(\varphi, \tau)$, and $f_{l_{C}}(\varphi, \tau)$, whereas Figs. 6(j)-6(1) show the corresponding circular mean phase profiles $\stackrel{\circ}{\mu}_{l_{A}}(\tau), \stackrel{\circ}{\mu}_{l_{B}}(\tau)$, and $\stackrel{\circ}{\mu}_{l_{C}}(\tau)$ (black curves) with their confidence intervals (gray regions).

To complete the phase decoding method, we define the mapping $\Psi$ in order to one-to-one associate any length $l=$ $\mu_{l}$ with the phase profile $\stackrel{\circ}{\mu}_{l}(\tau)$, with the related confidence interval:

$$
\Psi[l]=\stackrel{\circ}{\mu}_{l}(\tau), \quad \tau \in[0, l], l \in \Lambda, \stackrel{\circ}{\mu}_{l} \in \Pi .
$$


The uncertainty underlying this mapping is represented by another map $\Psi_{\sigma}$, which relates $l=\mu_{l}$ to the circular standard deviation profile $\circ_{l}^{\circ}(\tau)$ :

$$
\Psi_{\sigma}[l]=\circ_{\circ}^{\circ}(\tau), \quad l \in \Lambda, \stackrel{\circ}{\sigma}_{l} \in[0,1] .
$$

Figures 5(b) and 5(c) show the maps $\Psi$ and $\Psi_{\sigma}$ in the example considered.

\section{Part 3: Estimate of burst onset probability}

The last part of the method is a procedure that allows estimating burst onset probability for a generic input phase profile $\vartheta(\tau)$ starting at the beginning of an IBI. Let

$$
\vartheta_{l}(\tau)= \begin{cases}\vartheta(\tau) & \text { for } 0 \leqslant \tau \leqslant l \\ 0 & \text { otherwise }\end{cases}
$$

denote a part of $\vartheta(\tau)$, defined in the interval $[0, l]$. We want to estimate the probability of burst onset at $\tau=l$, given a phase profile $\vartheta_{l}$.

Let $\tilde{\varphi}_{l}$ be the phase profile that causes burst onset after an IBI length $l$, according to (9):

$$
\tilde{\varphi}_{l}(\tau)=\Psi[l], \quad \tau \in[0, l] .
$$

Since $\Phi$ is a metric space, we can always define the circular distance between $\vartheta_{l}(\tau)$ and $\tilde{\varphi}_{l}(\tau)$ [according to Eq. (E5)]:

$$
\mathcal{D}\left[\vartheta_{l}, \tilde{\varphi}_{l}\right]=\left|\sqrt{1-\frac{1}{2 l} \int_{0}^{l}\left|e^{j \vartheta_{l}(\tau)}+e^{j \tilde{\varphi}_{l}(\tau)}\right| d \tau}\right| .
$$

Hence $\mathcal{D} \in[0,1]$ and, for any length $l$, we can express the probability of burst onset at time $\tau$ for the neuron stimulated with an input with phase profile $\vartheta_{l}(\tau)$ as

$$
r_{l}(\tau)=1-\mathcal{D}\left[\vartheta_{l}, \tilde{\varphi}_{l}\right], \quad l \in \Lambda .
$$

Indeed, if $\mathcal{D}\left[\vartheta_{l}, \tilde{\varphi}_{l}\right]=1$ (maximum distance), the probability of burst onset at $\tau$ is $r_{l}(\tau)=0$, whereas if $\mathcal{D}\left[\vartheta_{l}, \tilde{\varphi}_{l}\right]=0$ (i.e., $\left.\vartheta_{l}=\tilde{\varphi}_{l}\right)$, the probability is $r_{l}(\tau)=1$. However, we have to take into account the uncertainty of the decoding process through the circular standard deviation profile $\Psi_{\sigma}[l]$, which is usually higher in the central part of the IBIs, as shown in Fig. 5(c).

In order to redefine the circular distance to account for this uncertainty, it is reasonable to give a higher weight to the distances $\mathcal{D}$ corresponding to the lowest values of $\stackrel{\circ}{\sigma}_{l}(\tau)$, since they correspond to the lowest uncertainty. The decoding reliability function $\chi_{l}:[0, l] \mapsto[0,1]$ is defined as follows, for any $\tau \in[0, l]$ :

$$
\chi_{l}(\tau)=1-\circ_{\circ}^{\circ}(\tau)
$$

Therefore, we can define a weighting function $\mathcal{W}_{l}(\tau)$, which represents the reliability of the probability of burst onset for any $\tau$, given $l$,

$$
\mathcal{W}_{l}(\tau)=\frac{\chi_{l}(\tau)}{\int_{0}^{l} \chi_{l}(\tau) d \tau}
$$

such that $\int_{0}^{l} \mathcal{W}_{l}(\tau) d \tau=1$. Then the weighted circular distance $\mathcal{D}_{\mathcal{W}} \in[0,1]$ can be defined as follows:

$$
\begin{aligned}
\mathcal{D}_{\mathcal{W}}\left[\vartheta_{l}, \tilde{\varphi}_{l}\right] & =\left|\sqrt{\int_{0}^{l}\left[1-\frac{1}{2}\left|e^{j \vartheta_{l}(\tau)}+e^{j \tilde{\varphi}_{l}(\tau)}\right|\right] \mathcal{W}_{l}(\tau) d \tau}\right| \\
& =\left|\sqrt{1-\frac{1}{2} \int_{0}^{l}\left|e^{j \vartheta_{l}(\tau)}+e^{j \tilde{\varphi}_{l}(\tau)}\right| \mathcal{W}_{l}(\tau) d \tau}\right| .
\end{aligned}
$$

Consequently, the phase-conditional time-dependent firing probability is redefined as follows:

$$
r_{l}(\tau)=1-\mathcal{D}_{\mathcal{W}}\left[\vartheta_{l}, \tilde{\varphi}_{l}\right] .
$$

Figure 7(a) shows the raster plot of $r_{l}(\tau)$ corresponding to the phase profiles $\varphi_{k}$ shown in Fig. 5(a). We notice that $r_{l}(\tau) \rightarrow 1$ for $\tau \rightarrow l$ and it is nonzero only for $\tau>\tilde{l}_{1}$ (minimum IBI length).

Observing phase profiles with $l>250 \mathrm{~ms}, \varphi(\tau)$ oscillates at least twice in $\Pi$. For instance, Fig. 7(b) shows two phase profiles corresponding to lengths $l_{a}$ (red curve) and $l_{b}$ (black curve): The similarity of the two profiles is apparent in the first $120 \mathrm{~ms}$, which justifies the yellow (high probability of burst onset) segment around this $\tau$ value in Fig. 7(a). More in general, a phase profile $\vartheta_{l}(\tau)$ with $l \in[120,200] \mathrm{ms}$ is associated with high values (greater than 0.6$)$ of $r_{l}(\tau)$; indeed, in this range the profile $\tilde{\varphi}_{l}(\tau)$ allows the burst onset also after a single phase oscillation.

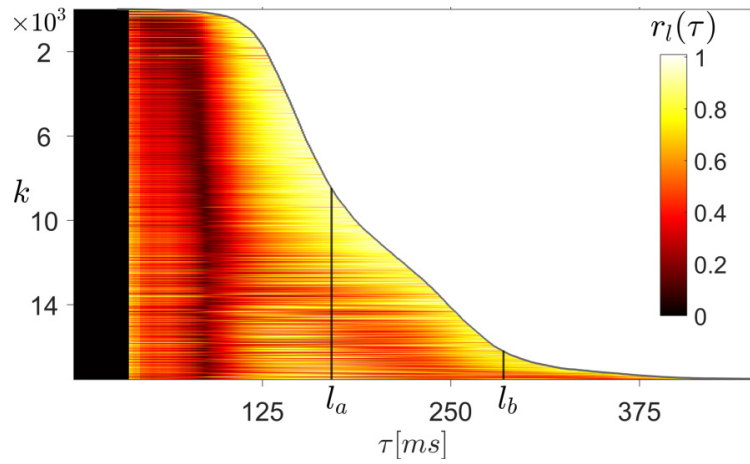

(a)

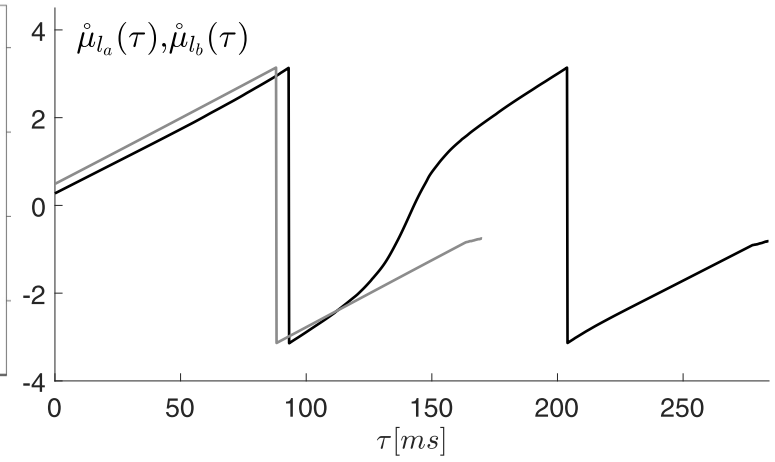

(b)

FIG. 7. (a) Raster plot of $r_{l}(\tau)$ corresponding to the phase profiles $\varphi_{k}(\tau)$ shown in Fig. 5(a); the black vertical segments mark two lengths $l_{a}$ and $l_{b}$. (b) Circular mean profiles of the phases corresponding to $l_{a}$ (gray curve) and $l_{b}$ (black curve). 


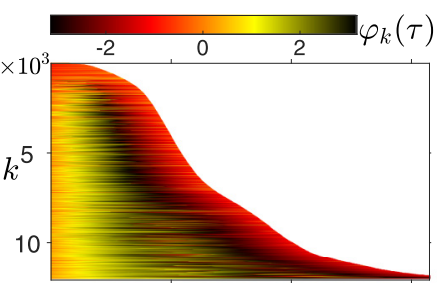

(a)

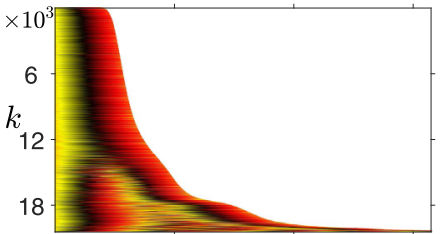

(e)

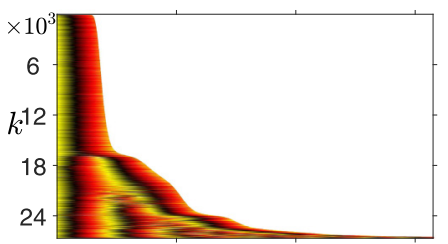

(i)

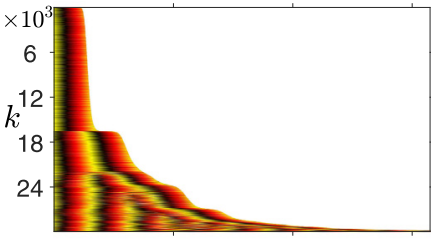

$(\mathrm{m})$

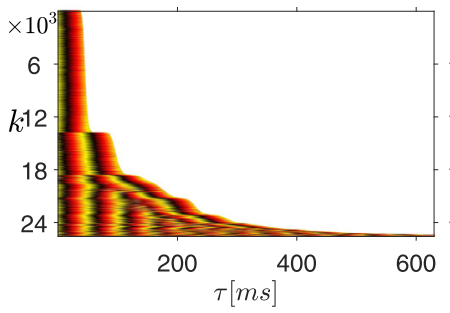

(q)

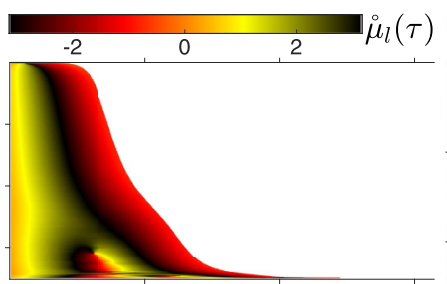

(b)

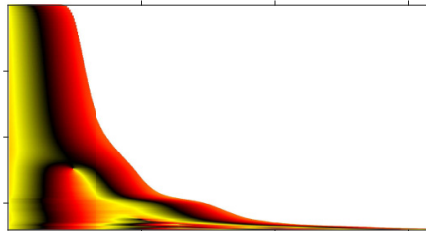

(f)

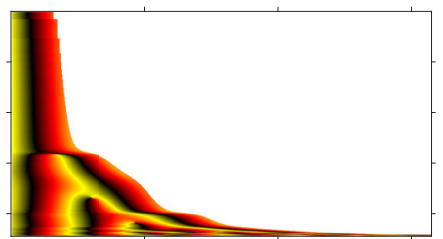

(j)

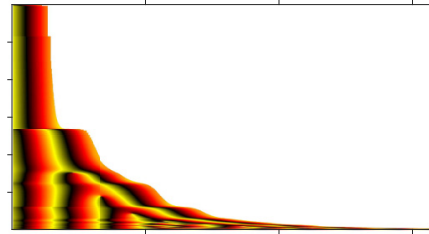

(n)

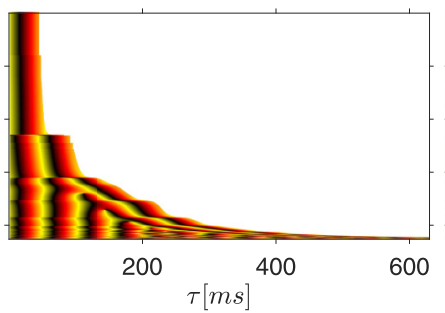

(r)

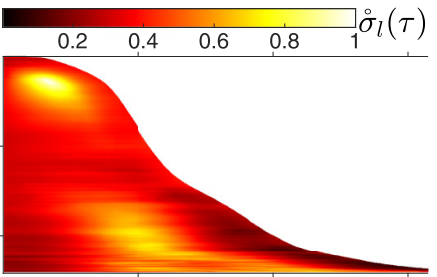

(c)

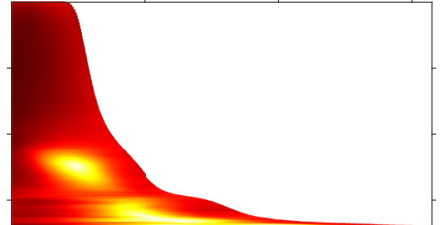

(g)

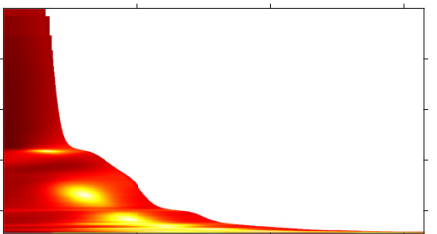

(k)

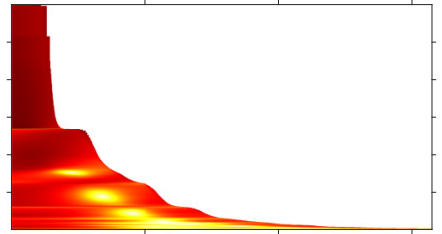

(o)

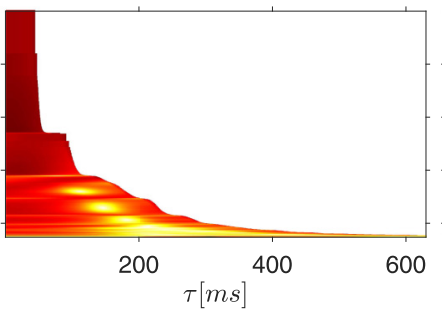

(s)

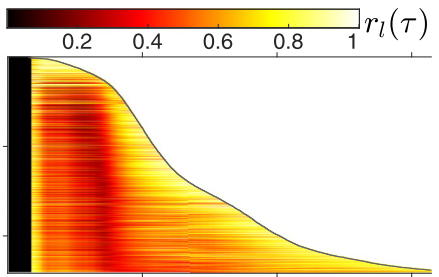

(d)

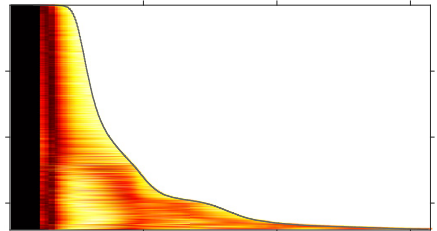

(h)

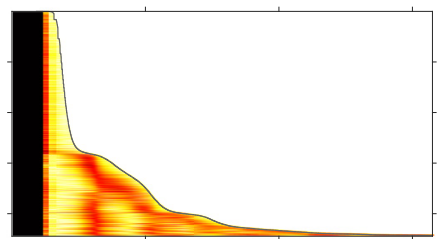

(1)

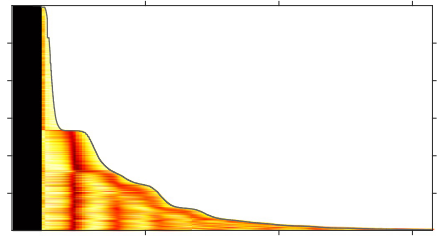

(p)

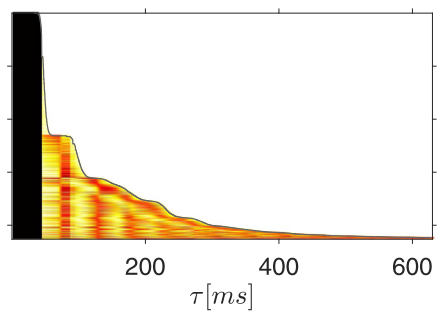

(t)

FIG. 8. Comparison of different kinds of raster plots (columns) for different frequency bands (rows). Raster plots of phase profiles $\varphi_{k}(\tau)$ (first column), circular mean phase profile $\stackrel{\leftrightarrow}{\mu}_{l}(\tau)$ (second column), circular standard deviation profile $\stackrel{\circ}{\sigma}_{l}(\tau)$ (third column), and phase-conditional firing probability $r_{l}(\tau)$ (fourth column) extracted from the neural response to stochastic stimulation with all four kinds of synaptic stimuli considered, filtered in the bands [1,5] Hz (first row), [5,9] Hz (second row), [9,13] Hz (third row), [13,17] Hz (fourth row), and [17,21] Hz (last row). Since the $\tau$ axis (in ms) is common to all figures and the $k$ axis is common to each row of figures, we show them only in the leftmost and bottom figures. The other figures keep only the axis ticks, as reference.

\section{RESULTS}

The proposed method has been tested with different inputs and neuron models. For the stochastic input, the parameters are detailed in Appendix B. The parameter $\varepsilon$ has been set to $15 \mathrm{~ms}$. The stimulation length is $1000 \mathrm{~s}$.

\section{A. Response to synaptic stimuli in other frequency bands}

First of all, we describe the results obtained by employing the pyramidal neuron model with filtered stochastic stimulations in partially overlapping frequency windows of bandwidth $4 \mathrm{~Hz}$, contained in the interval $[1,21] \mathrm{Hz}$. Panels in the first column of Fig. 8, from top to bottom, show raster plots of $\varphi_{k}(\tau)$, obtained [as in Fig. 5(a)] from the neural responses to stochastic stimuli in $[1,5],[5,9],[9,13],[13,17]$, and $[17,21] \mathrm{Hz}$ frequency bands, respectively. The corresponding maps $\Psi$ and $\Psi_{\sigma}$ are shown in the second and third columns, respectively, whereas panels in the last column show the corresponding phase-conditional time-dependent burst onset probability $r_{l}(\tau)$.

These results evidence the correlation between input phase profiles in different frequency bands and burst onset timing in the corresponding neural responses. As expected, for higher input frequencies the number of phase oscillations in $\Pi$ 


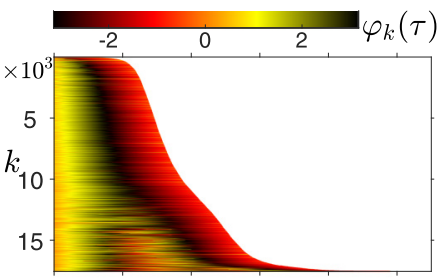

(a)

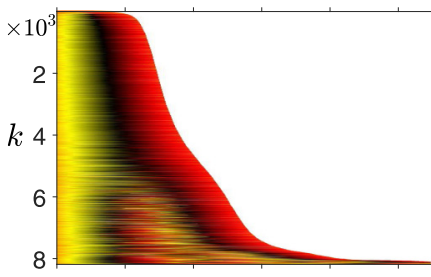

(e)

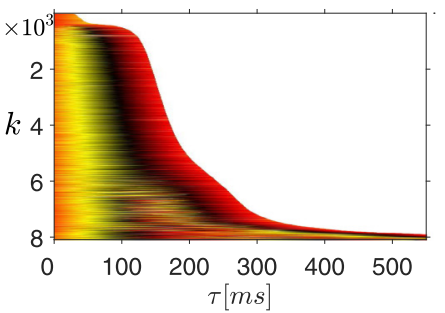

(i)

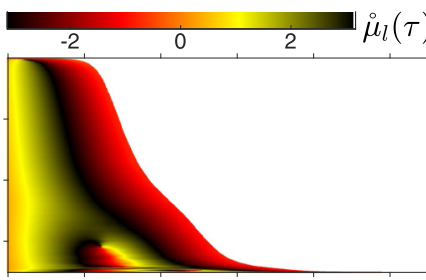

(b)

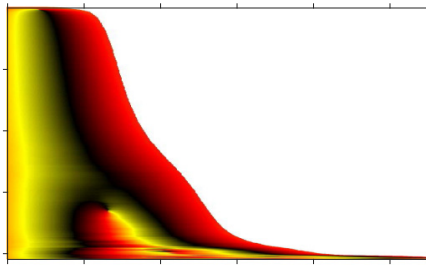

(f)

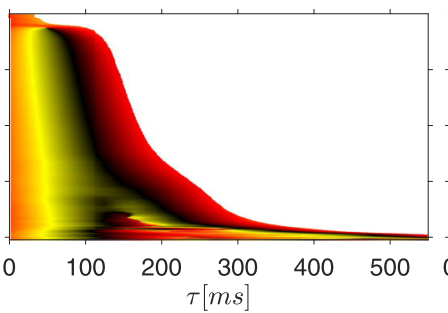

(j)

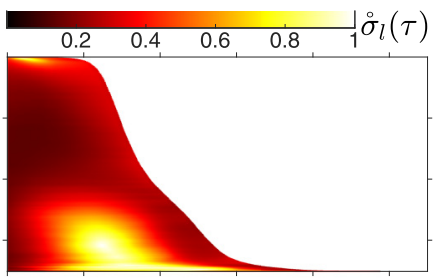

(c)

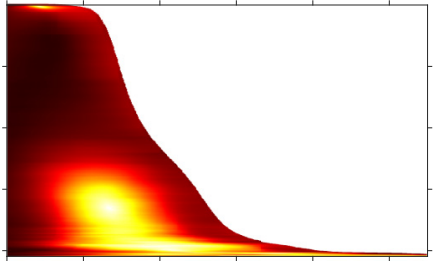

$(\mathrm{g})$

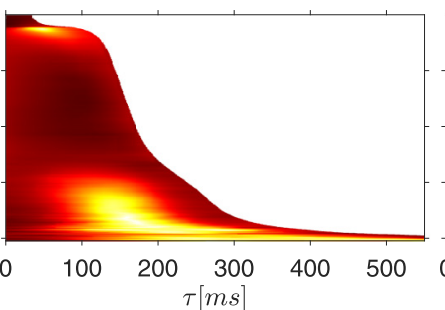

(k)

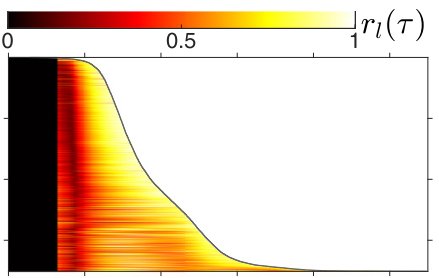

(d)

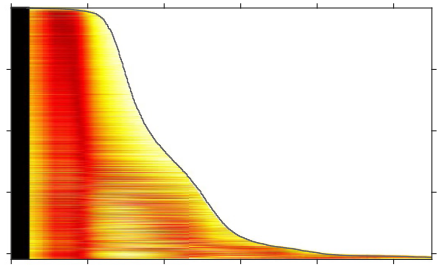

(h)

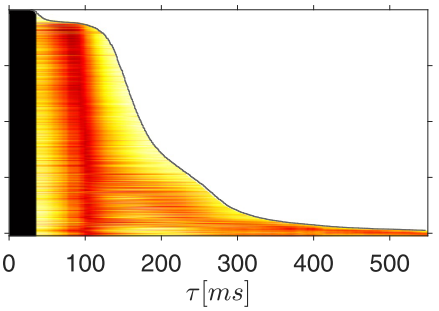

(1)

FIG. 9. Raster plot of $\varphi_{k}(\tau)$ (first column), $\stackrel{\circ}{\mu}_{l}(\tau)$ (second column), $\stackrel{\circ}{l}_{l}(\tau)$ (third column), and $r_{l}(\tau)$ (fourth column) extracted from the neural response to stochastic stimulation obtained by using all four kinds of synaptic stimuli considered, filtered in the bands [3,7] $\mathrm{Hz}$ for the pyramidal (first row), adaptive exponential integrate and fire (second row), and Izhikevich (third row) neuron models. See Fig. 8 caption about the axes.

(i.e., the number of oscillations needed to activate a burst) increases. Furthermore, highest values of decoding uncertainty are always located in the central part of the IBIs, i.e., far from bursts. The figure points out that the mean IBI value decreases (i.e., the burst are activated more frequently) by increasing the frequency band (it is $277 \mathrm{~ms}$ in [1,5] Hz, $171 \mathrm{~ms}$ in [5,9] Hz, $136 \mathrm{~ms}$ in $[9,13] \mathrm{Hz}, 124 \mathrm{~ms}$ in $[13,17] \mathrm{Hz}$, and $115 \mathrm{~ms}$ in $[17,21] \mathrm{Hz})$, even if the related energy decreases, as apparent from Figs. 2(e)-2(h).

As a first check of the method robustness, we repeated the same test employing two other neuron models: the adaptive exponential integrate and fire model [23] and the Izhikevich model [24]. By applying the same stochastic stimulations, we obtained results (shown in Fig. 9) very similar to those of Fig. 8, although these models are simpler than the pyramidal one.

\section{B. Response to sinusoidal synaptic stimulus}

As a second test, after identification of the maps $\Psi$ and $\Psi_{\sigma}$ related to the frequency window $[3,7] \mathrm{Hz}$, we attempted to reconstruct the phase profile of a pure sinusoidal input, which caused burst onset. The results are summarized in Fig. 10. Figure 10(a) shows a detail of the neural response to the synaptic input $I(t)=A \sin \left(2 \pi f_{0} t\right)$ [Fig. 10(b)] with amplitude $A=5 \mathrm{nA}$ and frequency $f_{0}=5 \mathrm{~Hz}$ (central value of the frequency band). The corresponding phase profile $\varphi_{I}(t)$ (detail) is shown in Fig. 10(c), where, like in Fig. 3, each black piece evidences a phase profile $\varphi_{k}$ that causes a burst onset; for this specific input, all the $\varphi_{k}(\tau)$ are identical, of length $L$. Then we can compute $\tilde{\varphi}_{L}(\tau)=\Psi[L]$, the phase profile of length $L$ that should cause a burst onset according to the decoding map $\Psi$. Figure $10(\mathrm{~d})$ shows $\tilde{\varphi}_{L}(\tau)$ and $\varphi_{k}(\tau)$. The two profiles are very similar, since, due to the circular symmetry, the phase lag can be neglected. Finally, Fig. 10(e) shows $r_{L}(\tau)$, computed by Eq. (18). As expected, $r_{L}(\tau)$ tends to 1 for $\tau$ tending to $L$.

\section{Response to up-down states}

As a final test, we checked if the proposed phase coding holds also for an up-down state input [18]. The input signal has two possible states: $A_{1}=0.5$ (up state) and $A_{0}=-0.5$ (down state). The conditional probabilities of transition have been set as follows: For the up state to down state transition they are 0.001 for the first $12 \mathrm{~ms}$ of the up state, 0.02 from 12 to $160 \mathrm{~ms}$, and then 0.25 ; for the down state to the up state transition they are 0.0003 for the first $12 \mathrm{~ms}$ of the down state, 0.003 from 12 to $55 \mathrm{~ms}$, and then 0.03 . To smooth the transitions, the obtained signal has been low-pass filtered with a FIR filter of order 300 and cutoff frequency of $4 \mathrm{~Hz}$.

Observing the phase profiles $\varphi_{k}$ that cause burst onset, one can notice that, when the synaptic input has two (e.g., from up to down and back to up) or more transitions, the time length needed to cause burst onset is shorter than when the input is always up; this is evident in Fig. 11(a), which shows a detail of the neuron response $V(t)$ (black line) to the corresponding up-down state input $I(t)$ (thick gray line). This suggests that burst onset timing in the considered model depends on the input phase profile and not on a simple integration of synaptic current 


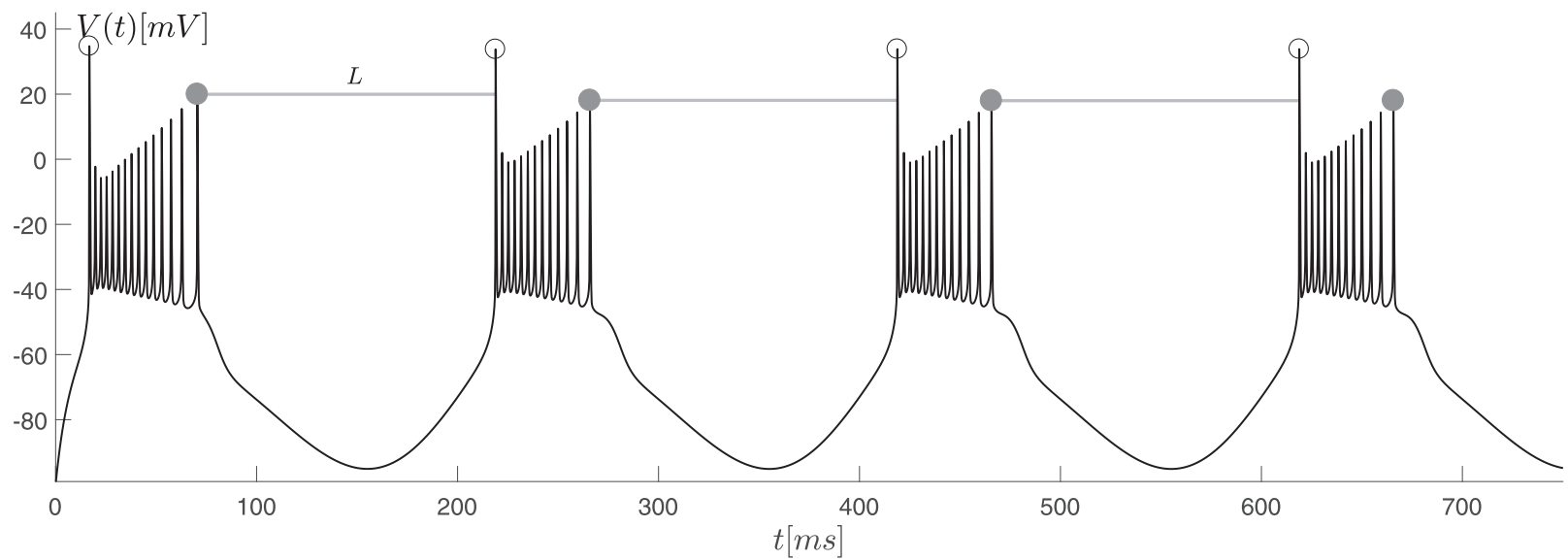

(a)

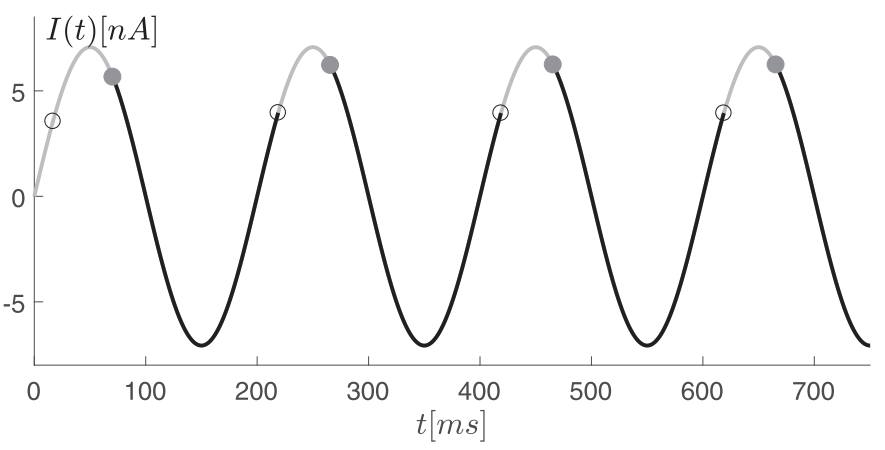

(b)

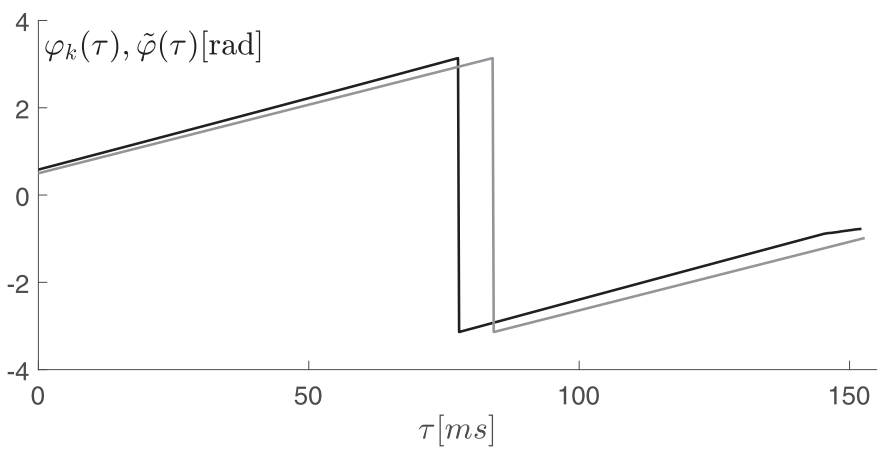

(d)

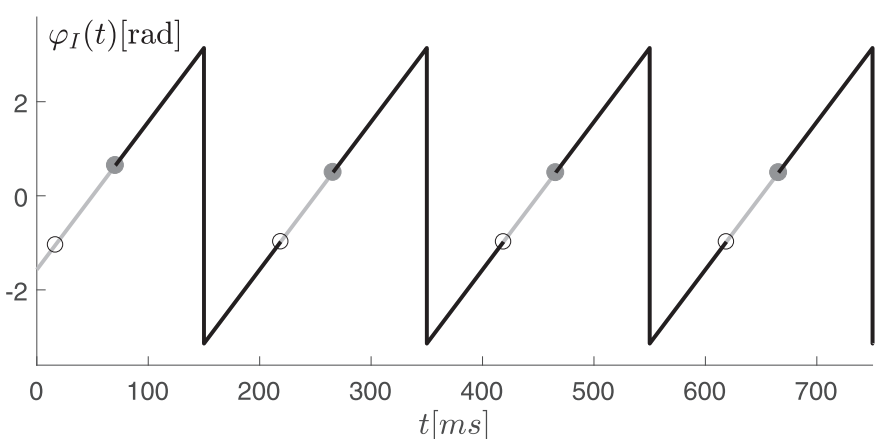

(c)

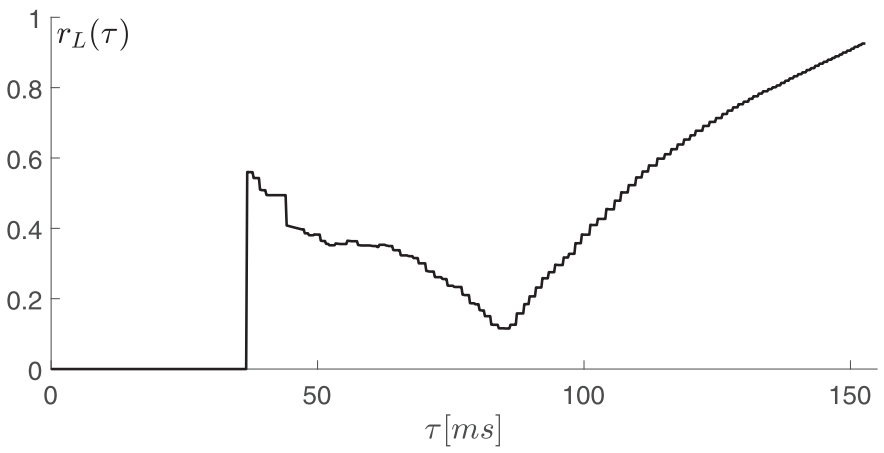

(e)

FIG. 10. Detail of the neural response [membrane potential $V(t)$ ] to (a) the synaptic input $I(t)$, corresponding to (b) the phase profile $\varphi_{I}(t)$. (c) Phase profiles $\varphi_{k}$ [zoom of $\left.\varphi_{I}(t)\right]$ and $\tilde{\varphi}_{L}(\tau)=\Psi[L]$. (d) Phase conditional time-dependent burst-onset probability $r_{l}(\tau)$.

(as for integrate-and-fire neuron models). Figures 11(b)-11(e) show the same plots $\left[\varphi_{k}, \Psi, \Psi_{\sigma}\right.$, and $r_{l}(\tau)$, respectively $]$ as in the other examples, related to phase coding and decoding of the considered up-down state input.

\section{DISCUSSION AND CONCLUSIONS}

The first result of this paper is a possible mechanism of phase coding relating the burst onsets of a neuron to the phase profile of the input current. Moreover, we defined the corresponding method of phase decoding and a procedure to estimate the likelihood of burst onset given the input state. Our results evidenced that the proposed methods provide good performances with neuron models of different kinds (the two-compartment pyramidal neuron model, the adaptive exponential integrate and fire model, and the Izhikevich model) and also with input stimuli of different nature (stochastic, sinusoidal, up, and down states). The chosen stochastic stimulation reproduces in the band $[1,21] \mathrm{Hz}$ the main typical features of low-frequency LFPs. In Sec. III C we simulated a neuron in a dynamic regime exhibiting up and down states, since this kind of activity involves large fluctuations of LFP and bursting activity [18] and is of obvious neurophysiological interest. However, we did not model any mechanism to originate such transitions (as was done, for instance, in $[25,26]$ ), but we simply designed an ad hoc stimulus to generate them. 


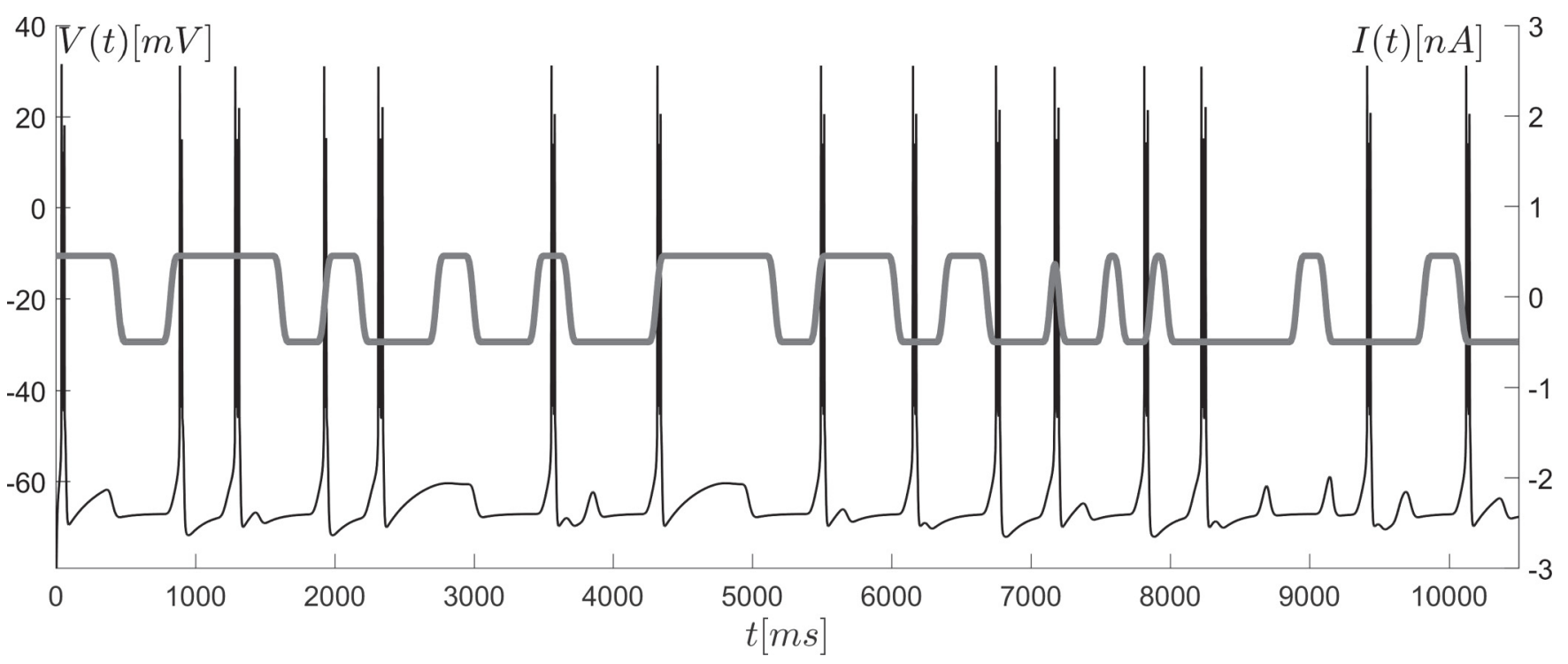

(a)

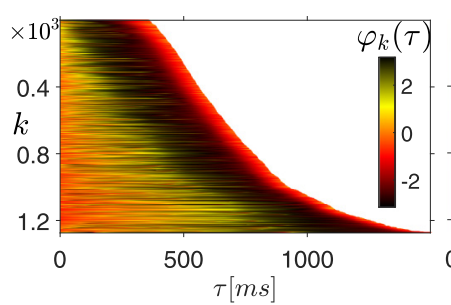

(b)

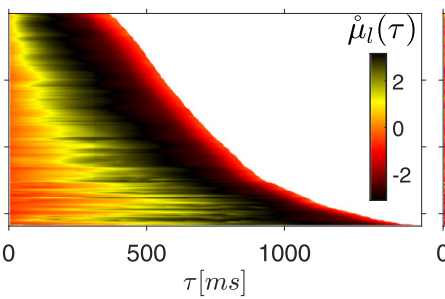

(c)

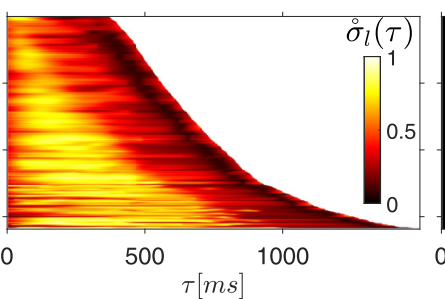

(d)

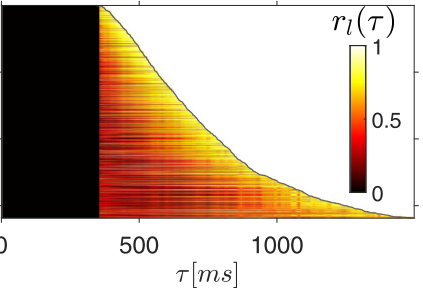

(e)

FIG. 11. (a) Detail of the input stimulus $I(t)$ (gray line, right axis) and corresponding membrane potential $V(t)$ (black line, left axis). Also shown are the raster plots of the phase profiles (b) $\varphi_{k}$, (c) $\stackrel{\circ}{\mu}_{l}(\tau)$, (d) $\stackrel{\circ}{\sigma}_{l}(\tau)$, and (e) $r_{l}(\tau)$ obtained from the complete stimulation (of length 1000 $\mathrm{s})$. Since the $k$ axis is common to (b)-(e), it is shown only in the leftmost figure.

\section{A. Limits of the proposed method}

The method proposed in this paper critically depends on some assumptions and parameters. The first assumption (related to the phase coding method) is about the power of the stimuli, which must be high enough to induce bursting, but not too high to have a good distribution of IBI lengths. In other words, the more output behaviors of the neuron are excited by the generated input stimuli, the more efficient the coding procedure is.

The second assumption (related to the phase decoding) is concerned with the one-to-one association between phase profiles and lengths, implying also that the IBI maximum and minimum lengths must be among those measured during the coding. This is of course related once more to the richness of the stimuli used in the coding method. The degree of fulfillment of this assumption is measured by $\Psi_{\sigma}$ : The more fulfilled the assumption, the lower the circular variance.

About the parameters, the most critical to be set are $\varepsilon$ (see Sec. II B) and $\sigma_{x}$ (see Appendix B). The parameter $\varepsilon$ defines the amplitude of the subinterval $\lambda_{l}$ and then influences the statistics of the functional subspace $\phi_{l}$. By increasing $\varepsilon$, we increase the number of lengths $\tilde{l}_{k} \in \lambda_{l}$, thus inducing both a smoother circular mean $\dot{\mu}_{l}(\tau)$ and a larger circular variance $\circ_{\sigma_{l}}(\tau)$, i.e., a larger uncertainty in estimating the burst onset probability. The chosen value of $\varepsilon=15 \mathrm{~ms}$ turned out to be a good trade-off between smoothness of map $\Psi$ and limited uncertainty in map $\Psi_{\sigma}$.

The parameter $\sigma_{x}$ is the standard deviation of the stochastic stimulus and influences its power. Consequently, $\sigma_{x}$ influences the IBI lengths: By decreasing $\sigma_{x}$, the upper limit of $\Lambda$ becomes larger due to the presence of longer IBIs, which are associated with higher uncertainties. The chosen values of $\sigma_{x}$ turned out to be a good trade-off to both induce burst onset and have IBI lengths lower than $600 \mathrm{~ms}$ in most cases (and then not to high uncertainty in the map $\Psi_{\sigma}$ ).

\section{B. Comparisons with previous works}

In [16] the authors correlate the phase value of the input stimulus at the burst onset with the number of spikes per burst, i.e., they propose a spike-count-based coding. In this paper, on the contrary, the coding is based on the phase profiles causing the burst onset. In other words, the phase coding mechanism proposed in this paper is based on phase pattern matching, rather than on punctual phase information.

In [7] it was suggested that, in sensory coding through LFPs, phase plays a role more important than energy in terms of information coding. This is the core of our method, where phase profiles play a key role. Indeed, how the information contained in the phase of the LFP could be transmitted is still an open question. Even phase-of-firing codes assume that 
neurons receiving spikes are somehow aware of the LFP phase at which they were emitted. Here we showed how a robust and reliable association between input phase and burst onset can be identified, hence we propose that burst activity could be an effective way to inform postsynaptic neurons of the current phase of the LFP.

\section{Neurophysiological validation and application}

We adopted here the model of spiking neuron introduced in [21], which is an efficient model of bursting neuron. To verify and possibly refine the model the following neurophysiological test could be designed: By injecting (in isolated neurons) current with selected spectral content while recording the membrane potential (and hence the spiking activity), the relationship between LFP phase and burst onset can be characterized and compared with the results presented here. Model parameters could then be tuned to match the bursting behavior of specific neuron types. The application of our results to neurophysiological studies is instead straightforward when investigating bursting dynamics in vivo with extracellular recordings. From an extracellular recording both LFP and spiking activity can be extracted [2,27]. In standard conditions, LFP can be considered a first-order approximation of the average input to the local neurons [27,28], even if the set of neural dynamics contributing to LFP is much more complex [4]. Consequently, from a single extracellular recording both the phase of the input and the bursting activity can be identified and combined to derive a phenomenological description of phase coding mechanisms as described in Sec. III.

\section{ACKNOWLEDGMENTS}

This work was partially supported by the University of Genoa. A.M. was supported by the internal fund of Scuola Superiore Sant'Anna Grant No. IEXERC14AM and by the Ministero degli Affari Esteri e della Cooperazione Internazionale, via the Italy-Sweden bilateral research project SE14GR4.

\section{APPENDIX A: PYRAMIDAL NEURON MODEL}

The considered pyramidal neuron model [21] is governed by the state equations

$$
C_{m} \frac{d V}{d t}=-I_{L}-I_{\mathrm{K}}-I_{\mathrm{Na}}-\frac{g_{c}\left(V-V_{d}\right)}{p}
$$

for the action potential $V$ of the soma compartment and

$$
C_{m} \frac{d V_{d}}{d t}=-I_{L}-I_{\mathrm{K} S}-I_{\mathrm{Na} P}-\frac{g_{c}\left(V_{d}-V\right)}{(1-p)}+I(t)
$$

for the action potential $V_{d}$ of the dendrite compartment. The ionic currents are

$$
\begin{gathered}
I_{\mathrm{Na}}=g_{\mathrm{Na}} m_{\infty}^{3} h\left(V-E_{\mathrm{Na}}\right), \\
I_{\mathrm{K}}=g_{\mathrm{K}} n^{4}\left(V-E_{\mathrm{K}}\right), \\
I_{\mathrm{Na} P}=g_{\mathrm{Na} P} r_{\infty}^{3}\left(V_{d}-E_{\mathrm{Na}}\right), \\
I_{\mathrm{K} S}=g_{\mathrm{K} S} q\left(V_{d}-E_{\mathrm{K}}\right), \\
I_{L}=g_{L}\left(V-E_{L}\right),
\end{gathered}
$$

where $I(t)$ is the synaptic stimulus. The gating variables are governed by the following kinetic equations:

$$
\begin{gathered}
\frac{d h}{d t}=\phi_{h}\left[\alpha_{h}(1-h)-h \beta_{h}\right] \\
\frac{d n}{d t}=\phi_{n}\left[\alpha_{n}(1-n)-n \beta_{n}\right] \\
\frac{d q}{d t}=\frac{q_{\infty}-q}{\tau_{q}}
\end{gathered}
$$

where

$$
\begin{gathered}
m_{\infty}=\frac{\alpha_{m}}{\alpha_{m}+\beta_{m}}, \\
r_{\infty}=\frac{1}{1+e^{-\left(V_{d}+57.7\right) / 7.7}}, \\
q_{\infty}=\frac{1}{1+e^{-\left(V_{d}+35\right) / 6.5}}, \\
\tau_{q}=\frac{\tau_{q 0}}{e^{-\left(V_{d}+55\right) / 30}+e^{\left(V_{d}+55\right) / 30}} .
\end{gathered}
$$

The kinetic coefficients are

$$
\begin{gathered}
\alpha_{m}=\frac{-0.1(V+31)}{e^{-0.1(V+31)}-1}, \\
\beta_{m}=4 e^{-(V+56) / 18}, \\
\alpha_{h}=0.07 e^{-(V+47) / 20}, \\
\beta_{h}=\frac{1}{e^{-0.1(V+17)}+1}, \\
\alpha_{n}=\frac{-0.01(V+34)}{e^{-0.1(V+34)}-1}, \\
\beta_{n}=0.125 e^{-(V+44) / 80} .
\end{gathered}
$$

The chosen model parameters are shown in Table I, where $g_{L}$, $g_{\mathrm{Na}}, g_{\mathrm{K}}, g_{\mathrm{Na} P}$, and $g_{\mathrm{K} S}$ are maximum conductances; $g_{c}$ is the coupling conductance connecting the two compartments; $E_{k}$, $E_{L}$, and $E_{\mathrm{Na}}$ are the reversal potentials; $C_{m}$ is the membrane capacitance; $\phi_{m}$ and $\phi_{n}$ are temperature scaling factors; $p$ is the relative area between the somatic and dendritic compartments;

TABLE I. Parameters of the pyramidal neuron model.

\begin{tabular}{lc}
\hline \hline Name & Value \\
\hline$g_{L}$ & $0.18 \mathrm{mS} / \mathrm{cm}^{2}$ \\
$g_{\mathrm{Na}}$ & $45 \mathrm{mS} / \mathrm{cm}^{2}$ \\
$g_{\mathrm{K}}$ & $20 \mathrm{mS} / \mathrm{cm}^{2}$ \\
$g_{\mathrm{Na} P}$ & $0.12 \mathrm{mS} / \mathrm{cm}^{2}$ \\
$g_{\mathrm{K} S}$ & $0.8 \mathrm{mS} / \mathrm{cm}^{2}$ \\
$g_{c}$ & $1 \mathrm{mS} / \mathrm{cm}^{2}$ \\
$E_{k}$ & $-90 \mathrm{mV}$ \\
$E_{L}$ & $-65 \mathrm{mV}$ \\
$E_{\mathrm{Na}}$ & $55 \mathrm{mV}$ \\
$C_{m}$ & $1 \mu \mathrm{F} / \mathrm{cm}^{2}$ \\
$\phi_{n}$ & 3.33 \\
$\phi_{h}$ & 3.33 \\
$p$ & 0.15 \\
$\tau_{q 0}$ & $250 \mathrm{~ms}$ \\
\hline \hline
\end{tabular}


and $\tau_{q 0}$ is a time constant, which influences the lifetime of bursts.

\section{APPENDIX B: STOCHASTIC PROCESSES}

The methods to generate realizations of the four considered stochastic processes are briefly summarized here.

White noise. The white noise $x_{w}(t)$ has been generated as a realization $N_{t}$ drawn at time $t$ from a Gaussian random variable with null mean and unitary variance $N(0,1)$.

Pink noise. Since the PSD $S_{p}(f)$ of pink noise must be proportional to $\frac{1}{f^{\alpha}}$ (with $0<\alpha<2$ ), a pink noise realization $x_{p}(t)$ can be obtained from a white noise realization $x_{w}(t)$ as

$$
x_{p}(t)=\mathcal{F}^{-1}\left\{\frac{1}{\sqrt{f^{\alpha}}} \mathcal{F}\left\{x_{w}(t)\right\}\right\},
$$

where $\mathcal{F}$ is the Fourier transform operator and we fixed $\alpha=1$.

Brown noise. The brown noise (or Brownian noise) realization $x_{b}(t)$ has been generated as

$$
x_{b}(t)=\int d W_{t},
$$

i.e., as the integral of the Wiener increment

$$
d W_{t}=W_{t+d t}-W_{t} \simeq \sqrt{d t} N(0,1),
$$

which approximates a Gaussian distribution with zero mean and variance $d t$. The PSD $S_{b}(f)$ of $x_{b}(t)$ is proportional to $\frac{1}{f^{2}}$, as shown in Fig. 2(d).

Ornstein noise. An Ornstein noise realization $x_{o}(t)$ has been computed by solving the SDE (Ornstein-Uhlenbeck process)

$$
d x_{t}=\theta\left(\mu-x_{t}\right) d t+\sigma d W_{t}
$$

through the Eulero-Maruyama method [29], with $\theta=0.05$, $\mu=1.2, \sigma=0.3$, and $d W_{t}$ defined in Eq. (B3).

Stochastic stimulus. Let $x_{r}(t)$ be the generic realization of one of the four processes described above $(r \in\{w, p, b, o\})$. Then we computed the stochastic stimulus $x(t)$, with zero mean and standard deviation $\sigma_{x}$, by applying the scaling

$$
x(t)=\sigma_{x} \frac{x_{r}(t)-\left\langle x_{r}(t)\right\rangle}{\sqrt{\left\langle x_{r}^{2}(t)\right\rangle-\left\langle x_{r}(t)\right\rangle^{2}}},
$$

where $\langle\cdot\rangle$ denotes the mean value (over a given interval) of the argument function. Since the brown noise has almost all power concentrated in the neighborhood of $f=0$, we fixed $\sigma_{x}=10$ for $r \in\{w, p, o\}$ and $\sigma_{x}=300$ for the brown noise term, in order to have comparable energies for the four signals in the frequency band $[1,21] \mathrm{Hz}$.

The $M$-length $\left(M=2 \times 10^{5}\right)$ discrete versions of the described signals have been obtained by sampling $x_{w}(t), x_{p}(t)$, $x_{b}(t)$, and $x_{w}(t)$ with step $\Delta t=5 \mathrm{~ms}$. Therefore, the total time length of $x(t)$ is $M \Delta t=10^{6} \mathrm{~ms}$.

\section{APPENDIX C: POWER SPECTRAL DENSITY OF STOCHASTIC PROCESSES}

The PSD $S_{x}(f)$ of a generic stochastic process $x(t)$ is defined as

$$
S_{x}(f)=\lim _{T_{w} \mapsto \infty} E\left\{\left|\frac{1}{\sqrt{T_{w}}} \int_{-T_{w} / 2}^{T_{w} / 2} x(t) e^{j 2 \pi f t} d t\right|^{2}\right\}_{x},
$$

where $T_{w}$ is a time window and $E$ is the mean over realizations of $x(t)$. We estimated $S_{x}(f)$ by using 700 realizations of length $T_{w}=14 \mathrm{~s}$.

\section{APPENDIX D: PHASE PROFILE DERIVATION}

The complex envelope $\tilde{I}(t)$ of a synaptic stimulus $I(t)$ is defined as

$$
\tilde{I}(t)=I(t)+j I_{Q}(t)=\rho(t) e^{j \varphi_{I}(t)},
$$

where $\rho(t)$ and $\varphi_{I}(t)$ are the modulus and phase of $\tilde{I}(t)$ and $I_{Q}(t)$ is the quadrature component of $I(t)$, computed by using the Hilbert transform $\mathcal{H}$ :

$$
I_{Q}(t)=\mathcal{H}\{I(t)\}=\frac{1}{\pi} \mathrm{P} . \mathrm{V} . \int_{-\infty}^{+\infty} \frac{I(t)}{t-\tau} d t .
$$

The phase profile $\varphi_{I}(t)$ (whose values are defined in the circular space $\Pi:[-\pi, \pi])$ is then computed as $\arg [\tilde{I}(t)]$.

\section{APPENDIX E: CIRCULAR STATISTICS}

In this appendix we briefly recall the elements of circular (or directional) statistics [30] necessary to compute the circular phase of the synaptic inputs. Let $\Pi:[-\pi, \pi]$ be the circular domain of the random variable $\theta$ with circular PDF $\stackrel{f}{f}(\theta)$. Then we can define the following quantities.

(i) The circular mean of $\theta$ is

$$
\stackrel{\leftrightarrow}{\mu}=\arg \left[\int_{\Pi} \stackrel{\circ}{f}(\theta) e^{j \theta} d \theta\right],
$$

where arg extracts the argument of a complex number and $e^{j \theta}$ uniquely identifies a specific point on the unit circle, for any $\theta \in \Pi$.

(ii) The circular distance between two values $\theta_{x}, \theta_{y} \in \Pi$ is

$$
{\stackrel{\circ}{\theta_{x}, \theta_{y}}}=\sqrt{1-\frac{1}{2}\left|e^{j \theta_{x}}+e^{j \theta_{y}}\right|},
$$

with ${\stackrel{\circ}{\theta_{x}, \theta_{y}}} \in[0,1]$. Owing to this definition, ${\stackrel{\circ}{\theta_{x}, \theta_{y}}}=0$ if $e^{j \theta_{x}}=$ $e^{j \theta_{y}}$ (i.e., $\theta_{x}$ and $\theta_{y}$ are the same element of $\Pi$ ) and ${\stackrel{\circ}{d_{x}, \theta_{y}}}=1$ if $e^{j \theta_{x}}=-e^{j \theta_{y}}$ (i.e., the phase shift $|\Delta \theta|=\left|\theta_{y}-\theta_{x}\right|=\pi$ on $\Pi$ is maximum).

(iii) The circular variance (which quantifies the meansquare deviation in $\Pi$ ) is

$$
\stackrel{\circ}{ }^{2}=1-\left|\int_{\Pi} \stackrel{\circ}{f}(\theta) e^{j \theta} d \theta\right| .
$$

(iv) Since

$0 \leqslant\left|\int_{\Pi} \stackrel{\circ}{f}(\theta) e^{j \theta} d \theta\right| \leqslant \int_{\Pi}|\stackrel{\circ}{f}(\theta)|\left|e^{j \theta}\right| d \theta=\int_{\Pi} f(\theta) d \theta=1$,

the circular variance $\stackrel{\circ}{ }^{2}$ ranges in the interval $[0,1]$, which allows defining the confidence interval of $\theta$ in $\Pi$ as $[\stackrel{\circ}{\mu}-\pi \stackrel{\circ}{\circ}, \dot{\mu}+\pi \stackrel{\circ}{\sigma}]$.

(v) Let $\Theta=\{\theta: \mathcal{I} \longmapsto \Pi, \mathcal{I} \subset \mathbb{R}\}$ be the functional space containing stochastic processes $\theta(t)$ with circular codomain $\Pi$, characterized by the PDF $f(\theta, t)$. Then we can compute first-order time-variant statistics $\stackrel{\circ}{\mu}(t)$ and $\stackrel{\circ}{ }^{2}(t)$ from (E1) and (E3), by replacing $\stackrel{\circ}{f}(\theta)$ with $\stackrel{f}{f}(\theta, t)$. Since $\Theta$ is a metric 
space, we can always define the circular distance $\mathcal{D}: \Phi \longmapsto[0,1]$ between two generic realizations $\theta_{x}(t), \theta_{y}(t) \in \Theta$ with $t \in[a, b] \subseteq \mathbb{R}$ by integrating over time the circular distance

$$
\mathcal{D}\left[\theta_{x}, \theta_{y}\right]=\sqrt{\left\langle{\stackrel{\circ}{\theta_{x}}}^{2}, \theta_{y}(t)\right\rangle}=\sqrt{1-\frac{1}{2(b-a)} \int_{a}^{b}\left|e^{j \theta_{x}(t)}+e^{j \theta_{y}(t)}\right| d t} .
$$

[1] G. Buzsaki, Rhythms of the Brain (Oxford University Press, Oxford, 2006).

[2] R. Q. Quiroga and S. Panzeri, Nat. Rev. Neurosci. 10, 173 (2009).

[3] P. Fries, Trends Cognitive Sci. 9, 474 (2005).

[4] G. Buzsáki, C. A. Anastassiou, and C. Koch, Nat. Rev. Neurosci. 13, 407 (2012).

[5] J. O'Keefe and N. Burgess, Hippocampus 15, 853 (2005).

[6] M. Mehta, A. Lee, and M. Wilson, Nature (London) 417, 741 (2002).

[7] C. Kayser, M. A. Montemurro, N. K. Logothetis, and S. Panzeri, Neuron 61, 597 (2009).

[8] M. A. Montemurro, M. J. Rasch, Y. Murayama, N. K. Logothetis, and S. Panzeri, Curr. Biol. 18, 375 (2008).

[9] C. Kayser, N. K. Logothetis, and S. Panzeri, Proc. Natl. Acad. Sci. USA 107, 16976 (2010).

[10] T. Masquelier, E. Hugues, G. Deco, and S. J. Thorpe, J. Neurosci. 29, 13484 (2009).

[11] A. Mazzoni, N. Brunel, S. Cavallari, N. K. Logothetis, and S. Panzeri, J. Physiol. 105, 2 (2011).

[12] A. Hayar, S. Karnup, M. T. Shipley, and M. Ennis, J. Neurosci. 24, 1190 (2004).

[13] S. M. Sherman, Trends Neurosci. 24, 122 (2001).

[14] J. Cang and J. S. Isaacson, J. Neurosci. 23, 4108 (2003).

[15] M. L. Lőrincz, K. A. Kékesi, G. Juhász, V. Crunelli, and S. W. Hughes, Neuron 63, 683 (2009).
[16] I. Samengo and M. A. Montemurro, PLoS ONE 5, e9669 (2010).

[17] D. H. Elijah, I. Samengo, and M. A. Montemurro, Front. Comput. Neurosci. 9, 113 (2015).

[18] Y. Shu, A. Hasenstaub, and D. A. McCormick, Nature (London) 423, 288 (2003).

[19] C. Curto, S. Sakata, S. Marguet, V. Itskov, and K. D. Harris, J. Neurosci. 29, 10600 (2009).

[20] A. B. Saleem, P. Chadderton, J. Apergis-Schoute, K. D. Harris, and S. R. Schultz, J. Comput. Neurosci. 29, 49 (2010).

[21] P. F. Pinsky and J. Rinzel, J. Comput. Neurosci. 1, 39 (1994).

[22] A. Kepecs, X.-J. Wang, and J. Lisman, J. Neurosci. 22, 9053 (2002).

[23] R. Brette and W. Gerstner, J. Neurophysiol. 94, 3637 (2005).

[24] E. M. Izhikevich et al., IEEE Trans. Neural Networks 14, 1569 (2003).

[25] A. Compte, M. V. Sanchez-Vives, D. A. McCormick, and X.-J. Wang, J. Neurophysiol. 89, 2707 (2003).

[26] N. Parga and L. F. Abbott, Front. Neurosci. 1, 57 (2007).

[27] N. K. Logothetis, J. Neurosci. 23, 3963 (2003).

[28] A. Mazzoni, H. Lindén, H. Cuntz, A. Lansner, S. Panzeri, and G. T. Einevoll, PLoS Comput. Biol. 11, e1004584 (2015).

[29] P. E. Kloeden and E. Platen, Numerical Solution of Stochastic Differential Equations (Springer, Berlin, 1992).

[30] K. V. Mardia, Statistics of Directional Data (Academic, New York, 2014). 